\title{
Advances in infrared gradient refractive index (GRIN) materials: a review
}

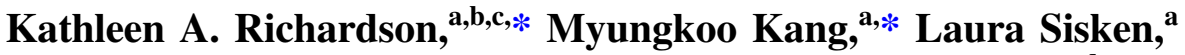 \\ Anupama Yadav, ${ }^{a}$ Spencer Novak, ${ }^{c}$ Antoine Lepicard, ${ }^{a, d}$ \\ Isabelle Martin, ${ }^{\mathrm{e}}$ Hugues Francois-Saint-Cyr, ${ }^{\mathrm{e}}$ Casey M. Schwarz, ${ }^{\mathrm{a}}$ \\ Theresa S. Mayer, ${ }^{\mathrm{f}}$ Clara Rivero-Baleine, ${ }^{\mathrm{g}}$ Anthony J. Yee, ${ }^{\mathrm{h}}$ and \\ Ilya Mingareevi \\ ${ }^{a}$ University of Central Florida, College of Optics and Photonics, Orlando, \\ Florida, United States \\ ${ }^{\mathrm{b}}$ University of Central Florida, Department of Materials Science and Engineering, Orlando, \\ Florida, United States \\ ${ }^{\mathrm{c}}$ Clemson University, Department of Materials Science and Engineering, Clemson, \\ South Carolina, United States \\ ${ }^{\mathrm{d} U n i v e r s i t e ́ ~ d e ~ B o r d e a u x, ~ I n s t i t u t ~ d e s ~ S c i e n c e s ~ M o l e ́ c u l a i r e s, ~ T a l e n c e, ~ F r a n c e ~}$ \\ ${ }^{\mathrm{e}}$ CAMECA Instruments Inc., Madison, Wisconsin, United States \\ ${ }^{\text {f}}$ Pennsylvania State University, Department of Electrical Engineering, University Park, \\ Pennsylvania, United States \\ ${ }^{g}$ Lockheed Martin Corporation, Orlando, Florida, United States \\ ${ }^{\text {h}}$ University of Rochester, Institute of Optics, Rochester, New York, United States \\ iFlorida Institute of Technology, Department of Mechanical and Civil Engineering, \\ Melbourne, Florida, United States
}

\begin{abstract}
Optical materials capable of advanced functionality in the infrared will enable optical designs that can offer lightweight or small footprint solutions in both planar and bulk optical systems. The University of Central Florida's Glass Processing and Characterization Laboratory, together with our collaborators, have been evaluating compositional design and processing protocols for both bulk and film strategies employing multicomponent chalcogenide glasses (ChGs). These materials can be processed with broad compositional flexibility that allows tailoring of their transmission window, physical and optical properties, which allows them to be engineered for compatibility with other homogeneous amorphous or crystalline optical components. We review progress in forming ChG-based gradient refractive index (GRIN) materials from diverse processing methodologies, including solution-derived ChG layers, poled ChGs with gradient compositional and surface reactivity behavior, nanocomposite bulk ChGs and glass ceramics, and metalens structures realized through multiphoton lithography. We discussed current design and metrology tools that lend critical information to material design efforts to realize next-generation IR GRIN media for bulk or film applications. (C) 2020 Society of Photo-Optical Instrumentation Engineers (SPIE) [DOI: 10.1117/1.OE.59.11.112602]
\end{abstract}

Keywords: chalcogenide glass; infrared gradient refractive index; multiphoton lithography; poled chalcogenide glass; optical nanocomposites; glass ceramics; integrated photonics; gradient refractive index metrology.

Paper 20200319SSV received Mar. 18, 2020; accepted for publication May 19, 2020; published online Jun. 17, 2020.

\section{Introduction}

Multicomponent chalcogenide glass (ChG) compounds suitable for use in planar or bulk form have been prepared with the goal of creating advanced optical functionality beyond those of currently available homogeneous optical materials. Recent advances have demonstrated the

*Address all correspondenceto Kathleen A. Richardson, E-mail: kcr@creol.ucf.edu; Myungkoo Kang, E-mail: myungkoo@creol.ucf.edu 
ability to compositionally tune not only optical properties (spectral window, refractive index, dispersion, $d n / d T^{1-6}$ ), but also properties important to their bulk, planar film, or fiber optical manufacturing [such as thermal and chemical stability, ${ }^{7-9}$ coefficient of thermal expansion (CTE), microhardness, fracture toughness, postmolding relaxation behavior, ${ }^{10-12}$ and compatibility with anti-reflective coating protocols $].{ }^{13}$ Some of these attributes are especially important if the material is to be deployed in a planar photonic system where stability (to thermal decomposition, phase separation, and scattering) can define suitability for the application and/or limit the material's use in an optical design. Over the past decade, CREOL's Glass Processing and Characterization Laboratory team members, our collaborators and others have made notable advances in developing new material systems, demonstrating they can be scaled up beyond the laboratory scale with commercial partners, ${ }^{14}$ and most importantly, highlighting the flexibility in optical function enabled by the creation of low loss, manufacturing-process compatible compositions. ${ }^{15}$ These compositions and their applications are presented and reviewed here, with the key aspects and suitability for their use in diverse environments highlighted. Most specifically, examples where this process know-how has been applied to the creation of gradient refractive index (GRIN) applications in the IR spectral window are discussed. We include for completeness of our review reference to other advances in materials for IR GRIN. High-level details related to composition, process methodology, and optical performance flexibility along with fabrication limitations are presented. In addition, a short summary of recently reported design strategies to integrate properties of GRIN materials into optical designs, as well as progress in the development and capabilities of optical metrology tools specifically tailored to GRIN materials and their use on candidate IR GRIN materials, is included.

\section{IR GRIN Materials}

Over the course of the last decade, advances in materials research resulting in GRIN as well as gradients in other physical properties have been extended to the IR. These materials are attractive in areas such as color or aberration correction and offer possible advantages as compared to other diffractive or refractive optical approaches. ${ }^{16}$ Strategies developed for visible materials (glasses and polymers) such as ion exchange and lamination/interdiffusion ${ }^{17-24}$ have been attempted in the IR with varying levels of success. These technologies differ in their approaches and compatibility to large-scale manufacturability and are at varying levels of progress toward commercialization. Clearly, the interest in developing next-generation devices for commercial or defense applications will benefit from an ability to integrate optical functions into smaller, more compact packages. This process, enhancing SWAP-C (size, weight, performance/power, and cost) is the goal toward making lighter weight, smaller footprint systems. Most activities to date are examining the use of GRIN materials for the IR in passive (undoped) applications such as lenses or windows that do not require high (laser) power transmission where it is known that ChGs (typically evaluated in fiber form) do not perform well due to modest laser damage resistance and high optical nonlinearities. ${ }^{25-31}$ However, many of the strategies discussed herein are not limited to passive materials as long as the manufacturing methodology used to create the GRIN does not result in adverse effects to the dopant species that degrades optical performance. Typical types of degradation could include extensive crystal growth (scattering), dopant segregation and/or clustering (scatter and absorption loss), and or preferential diffusion of dopant species in protocols employing temperature-dependent processing (resulting in spatial inhomogeneity of the dopant). This review examines and comments on some of these issues, in the context of summarizing progress on candidate materials and strategies for IR GRIN.

Development of promising candidate GRIN materials and technologies in the IR has been limited but has seen recent interest from funding agencies and programs (such as DARPA's M-GRIN program, which ran from 2010 to $2016^{32}$ ). However, many advances that resulted from this program remain at the laboratory-scale or have advanced to prototype level in their production, still awaiting further investment for design-specific development. That said, such technologies are aggressively sought after, as legacy crystalline materials cannot easily be manipulated in the same way that glass and glass ceramic materials can, to offer tailorable/ 
Table 1 IR GRIN technologies, attributes, and possible limitations or opportunities.

\begin{tabular}{ll}
\hline \hline $\begin{array}{l}\text { Technology: B-bulk } \\
\text { or P-planar }\end{array}$ & $\begin{array}{l}\text { Key material and manufacturability } \\
\text { attributes }\end{array}$ \\
\hline $\begin{array}{l}\text { B: Laminated/ } \\
\text { interdiffused ChGs }\end{array}$ & $\begin{array}{l}\text { Multicomponent/index materials } \\
\text { are laminated and thermal } \\
\text { interdiffusion results in a } \\
\text { composition and refractive index } \\
\text { profile associated with chemical } \\
\text { mixing of glass constituents; no } \\
\text { hard interface results; axial index } \\
\text { modifications to } \Delta n=+0.4 \text { (as } \\
\text { measured in the NIR) have been } \\
\text { demonstrated. }\end{array}$ \\
$\begin{array}{ll}\text { B: Thermally poled } \\
\text { diffractive or }\end{array}$ & $\begin{array}{l}\text { Mobile ion-containing bulk glass is } \\
\text { refractive optics }\end{array}$ \\
& $\begin{array}{l}\text { subjected to a thermal poling } \\
\text { process (applied electric field and } \\
\left.\text { temperatures near } T_{g}\right) \text { to create a } \\
\text { composition gradient between } \\
\text { electrodes; electrode patterning } \\
\text { imparts gradient to composition } \\
\text { profile with arbitrary shape } \\
\text { (grating, microlens, etc.) without } \\
\text { transmission degradation. Index } \\
\text { changes }(\Delta n) \text { to }+0.05 \text { in ChG } \\
\text { shown with long-lived (>1.5 year) } \\
\text { stability. }\end{array}$ \\
&
\end{tabular}

$B$ and P: Thermally induced (furnace or laser-induced) crystallization to form glass ceramics

Glasses (wide composition space available) which can undergo spatially controlled nucleation and realize index modification beyond growth result in formation of a high that of base material. index, nanocrystalline phase within a low(er) index glassy matrix; gradient in volume fraction of crystallite leads to an effective refractive index change related to the volume fraction of crystal and its identify (refractive index). Nanocomposite GRIN structures (radial and/or axial) can be formed within a (linear) thermal gradient, via spatially defined nucleation and homogeneous (temperature) growth, or through homogeneous nucleation and spatially varying (laser-induced) growth.

Formation of a glass ceramic results in intermediate thermal/ mechanical properties. Tailoring the crystal phase (and its index) as well as the volume fraction defines the magnitude of index change $(\Delta n)$; observed $\Delta n$ is base composition dependent but ranges to 0.2 in the MWIR/LWIR.

$2 \pi$ phase shift possible with high index base glass and large (film or bulk layer) thickness. Thick films must be CTE matched to substrate to avoid delamination.

$B$ and $P$ : laserinduced vitrification (LIV)

P: Solution-derived (SD) printing
Glasses which have undergone controlled crystallization (nanocrystalline phase formation) are subjected to DLW which under specific irradiation conditions leads to reamorphization; return of localized regions to the glassy state leads to patterns of index variation.

ChG powder dissolved in amine solutions are "inks" which can be "printed" onto substrates via spin coating (for axial GRIN) or via electrospray (for arbitrary film profile GRIN). Index profile limited by parent glass index and ability to coat to desired thickness.
Laser writing parameters are material specific.

Within bulk or near-surface reamorphization is possible. Correlation of loss levels to irradiation conditions has not to date been optimized for all materials studied; diffractive elements have been fabrication but efficiency has not been quantified.

Intermediate ChG layers must be heat treated to remove IR absorbing solvent; adjacent layer must not be soluble in target layer solvent; solvent choice, heat treatment protocol, and solution viscosity require optimization for optical quality film formation. 
Table 1 (Continued).

\begin{tabular}{|c|c|c|c|}
\hline $\begin{array}{l}\text { Technology: B-bulk } \\
\text { or P-planar }\end{array}$ & $\begin{array}{l}\text { Key material and manufacturability } \\
\text { attributes }\end{array}$ & $\begin{array}{l}\text { Possible limitations or } \\
\text { opportunities }\end{array}$ & Reference \\
\hline $\begin{array}{l}\text { P: Chemical vapor } \\
\text { deposition (CVD) }\end{array}$ & $\begin{array}{l}\text { Layer-by-layer deposition process } \\
\text { (with varying gas fractions) creates } \\
\text { refractive index variation } \\
\text { proportional to fraction of parent } \\
\text { crystal phase in an IR transmissive } \\
\text { multiphase polycrystalline } \\
\text { material. Processing resulted in } \\
\text { thick films (up to) } t \sim 4 \mu \mathrm{m} \text { thick } \\
\text { with a } \Delta n \text { ranging from } 0.2 \text { to } 0.6 \\
\text { (measured at } \lambda=0.647 \mu \mathrm{m} \text { ). }\end{array}$ & $\begin{array}{l}\text { CVD codeposition of ZnS and } \\
\text { ZnSe to form a ZnSSe material } \\
\text { with a near-linear axial gradient. } \\
\text { Physical property variation } \\
\text { observed, with hardness (related } \\
\text { to grain size), intermediate } \\
\text { between the two end-points. } \\
\text { Transmission was inferior to } \\
\text { parent crystalline end points (ZnS } \\
\text { and ZnSe) but improved following } \\
\text { hot isostatic press processing. }\end{array}$ & 18 \\
\hline $\begin{array}{l}\text { P: Laser-induced } \\
\text { photopolymerization }\end{array}$ & $\begin{array}{l}\text { Photosensitive ChGs (select } \\
\text { compositions) are exposed to } \\
\text { near-bandgap light inducing } \\
\text { modification to cross-linking within } \\
\text { glass film network. This enables } \\
\text { etch selectivity between exposed } \\
\text { and unexposed regions. }\end{array}$ & $\begin{array}{l}\text { Limited to ChG compositions } \\
\text { which possess bonds that can } \\
\text { undergo spatially selective cross- } \\
\text { linking (or depolymerization) upon } \\
\text { exposure enabling patterning } \\
\text { which becomes etch selective. } \\
\text { Postexposure pattern fidelity } \\
\text { stability is composition specific. }\end{array}$ & $57-59$ \\
\hline
\end{tabular}

tunable optical properties, without expensive multimaterial coating and/or fabrication strategies that often degrade parent material optical quality. Following a short summary of the state-of-theart, we discuss some of the key attributes and possible limitations of ChG-based GRIN materials investigated by various researchers in this area. Key areas where these materials could benefit with further improvements possible with additional process development, enhanced optical design, and metrology tools that can work to more specifically define application-specific limitations are highlighted.

Table 1 reviews the current state-of-the-art for IR GRIN and summarizes each technology's suitability for bulk and/or planar applications. Not discussed here but contained in the references shown for these methodologies are specifics as to the GRIN formation process, the impact on magnitude, sign of resulting refractive index, and dispersion profiles over defined spectral ranges. As is known, precise design tools and characterization methodologies for GRIN materials in the IR are a technical challenge as metrology tools for this spectral region have often not been optimized for such components. ${ }^{60,61}$ A brief summary of progress in these two areas is included in Sec. 2.3. Included in the table are key attributes that define possible limitations or opportunities unique to the technologies. Here, data are reported based on published aspects/ values of performance for comparison. Specific attributes of key technologies developed by the authors and our collaborators are discussed in more detail below.

\subsection{Bulk GRIN Materials}

\subsubsection{GRIN by thermal poling}

Thermal poling of glass is a technology that has been known since the early $1990 \mathrm{~s}^{62-64}$ The process involves the application of an electric field across a specimen held at high temperature whereby mobile ions in the glass, usually alkali ions, can migrate in directions related to the induced field lines through the glass volume. This migration leads to the establishment of not only a localized depletion of alkali species, but also the freezing in of an induced space charge. The structural reorganization of the poled glass leads to the formation of a material that is not only chemically modified, but also modified in its linear and nonlinear optical properties. Whether this reorganization and the physical properties it imparts to the postpoled material is permanent or prone to relaxation depends on how the now displaced mobile cation can be "stabilized" or locked in to the now "modified" glass structure. 
Thermal poling of ChG materials has been known since the late 1990s as a means to induce optical property modification, ${ }^{65,66}$ but has suffered from short-term stability due to the low glass transition temperatures of these glasses and the relaxation of the postpoled structure with time, when aged near room temperature. Recently, researchers have demonstrated the ability to engineer the parent glass's network structure to enable long-lived postpoling-induced index change with tailorable magnitude and gradient shape through a nanoimprinting process. ${ }^{67}$ This result enables the use of surface-modified bulk ChGs as well as a variety of oxide glasses ${ }^{67-72}$ to realize induced second harmonic generation (SHG), linear refractive index changes, as well as modification to surface chemical reactivity.

Poling induces changes in physical properties to the local glass network, as shown in a sample specification sheet compiled for one glass composition (B3-P6: $\mathrm{Ge}_{22.5} \mathrm{Sb}_{10} \mathrm{~S}_{67.5}-3 \mathrm{~mol}$. \% $\mathrm{Na}_{2} \mathrm{~S}$ ) following a specific (singular) set of poling conditions (Fig. 1). Poling can change other physical properties of the glass (beyond the refractive index and dispersion properties) and optical designers who want to include such attributes in their designs require complete material property information. Hence, analysis of which properties are modified is critical to potential adoption of candidate glasses. This is why such data as shown in the specification sheet have been deemed valuable by personnel considering candidate materials. What is not discussed here but is discussed in detail in Refs. 39 and 73 is that these conditions can be varied to also change the shape of the postpoled alkali ion compositional profile, the local density, as well as the gradient in physical properties that results from the poling process. Key attributes for the modification correlate with a partial depletion of mobile alkali ions and the glass's response to this depletion from the anode surface. The magnitude of specific compositional changes can be quantified through secondary ion mass spectrometry (SIMS) analysis, as shown in Fig. 2(a). Figure 2(b) shows the optical transmission of the material through the IR. Most impressive is the fact that through compositional tuning of the parent glass believed to yield defective bonding configurations with a net negative charge, these species serve to stabilize the postpoling mobility of the $\mathrm{Na}^{+}$thereby "locking in" the induced properties over time. This provides needed charge compensation to the network and allows the resulting compositional gradient to be stable. The gradient in composition translates to a refractive index gradient that varies with the magnitude of alkali ion doping level but is long-lived upon aging at room temperature. The induced $\Delta n$ is stable over temperature excursions $\left(-40^{\circ} \mathrm{C}\right.$ to $\left.+80^{\circ} \mathrm{C}\right)$. By patterning the electrode associated with the poling process (i.e., to form circles, lines, or any other spatial feature shapes), this compositional profile can be imparted with specifically chosen shapes (i.e., microlenses or gratings) that can imprint optical function into the glass surface. The magnitude and gradient shape of the refractive index profile can be changed with variation in the electrode pattern shape and size. The measured refractive index stability (at $\lambda=4.5 \mu \mathrm{m}$ ) in a sample poled with an unpatterned, homogeneous ITO anode and cathode coating is shown in Fig. 3(a). Refractive index was monitored on the same sample under similar measurements conditions over the course of 1.5 years. The IR refractometer resolution is $5 \times 10^{-4}$ so the magnitude of the changes seen $(-0.047)$ is well above the sensitivity of the system. The minor variations seen from the dashed line meant to serve as a guide to the eye (i.e., data at 5 and 9 weeks) are likely due to minor local variations in measurement position and local chemistry (spot to spot measurement positions were not the same and thus depleted $\mathrm{Na}^{+}$levels and resulting network density could vary). While each of these points were measured by different personnel, which may have caused some variation, measured data fluctuation is likely true to the sample's surface modification, as data at 60 weeks are consistent with the earlier measurements. As can be seen, the stability imparted by the compositional tailoring of the glass matrix to stabilize the mobile $\mathrm{Na}^{+}$ions to postprocess migration leads to formation of a robust, permanent index gradient. This can be used to create spatially varying composition and index gradients as shown for the microlens array (MLA) shown in Fig. 3(b). As referred to above and reported in Ref. 39, the shape of this gradient (both its magnitude and slope) can be altered by modifying the patterned electrode's shape. It is also noteworthy to mention that currently available SCHOTT glass compositions are not amenable to GRIN as the current commercially available glass types do not contain sodium, which is a prerequisite for poling. In addition, the composition of the parent glass has been modified such that when poled, the glass's structure possesses sufficient bonding sites where the newly displaced alkali ion can be stabilized preventing relaxation of the induced index change. 


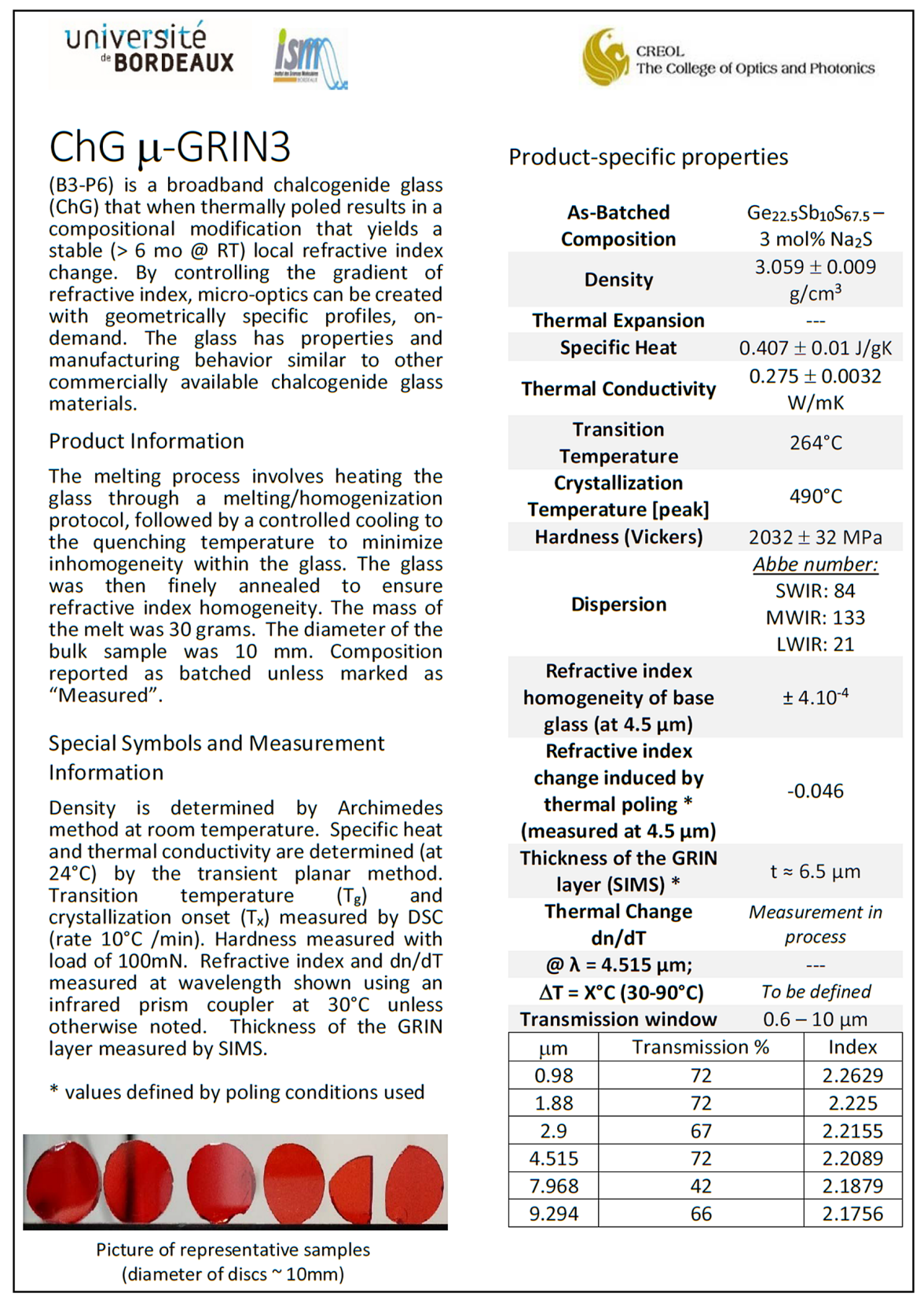

Fig. 1 Sample specification sheet for ChG $\mu$-GRIN3 GeSbS (3 mol. \% $\mathrm{Na}_{2} \mathrm{~S}$ ) glass summarizing characteristic material properties of the unpoled materials. Such data are critical to optical designers examining the potential of the candidate materials in their system designs.

\subsubsection{GRIN via spatially controlled nucleation and growth}

As part of DARPA's MGRIN program initiated in $2010^{32}$ and introduced by the Penn State-led team in our first presentation made at SPIE in $2016,{ }^{42}$ researchers demonstrated the ability to compositionally tailor multicomponent $\mathrm{ChGs}$ to create multiple glasses with varying refractive indices, which are amenable to controlled nucleation and growth of high index crystals within a low index matrix to create optical nanocomposites. The DARPA funded effort has focused on optimization of a $\mathrm{GeSe}_{2}-\mathrm{As}_{2} \mathrm{Se}_{3}-\mathrm{PbSe}(\mathrm{GAP}-\mathrm{Se})$ glass material for use in the midwave infrared (MWIR). In an optical glass ceramic nanocomposite, the effective properties result from the relative fractions and optical properties of both the glassy and crystalline phases present in the composite material. Exploiting fundamental glass science/crystallization behavior and 
(a)

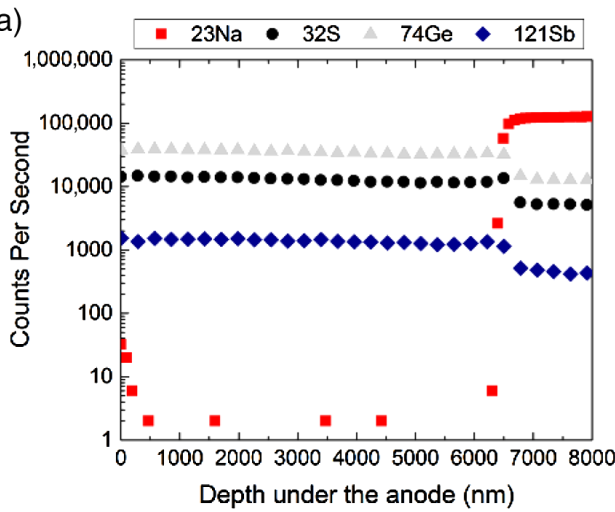

(b)

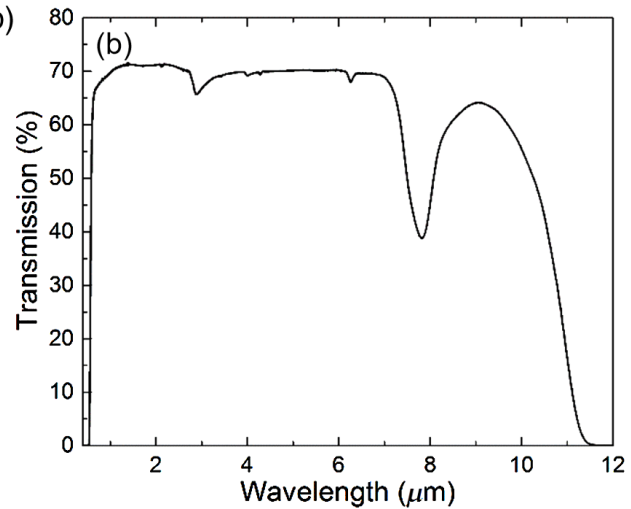

Fig. 2 (a) SIMS profile of the compositional variation induced by thermal poling. The signal decrease of $\mathrm{Ge}, \mathrm{Sb}$, and $\mathrm{S}$ at $\lambda=6.5 \mu \mathrm{m}$ is due to charging of the sample during the measurement, but illustrates the near-surface depletion of $\mathrm{Na}$ after poling. (b) Transmission spectrum (not corrected for Fresnel loss); sample thickness $=1.04 \mathrm{~mm}$ and sample diameter $=10 \mathrm{~mm}$ (as shown in Fig. 1). (a) Reproduced with permission from Ref. 38. Copyright 2018 OSA.

(a)

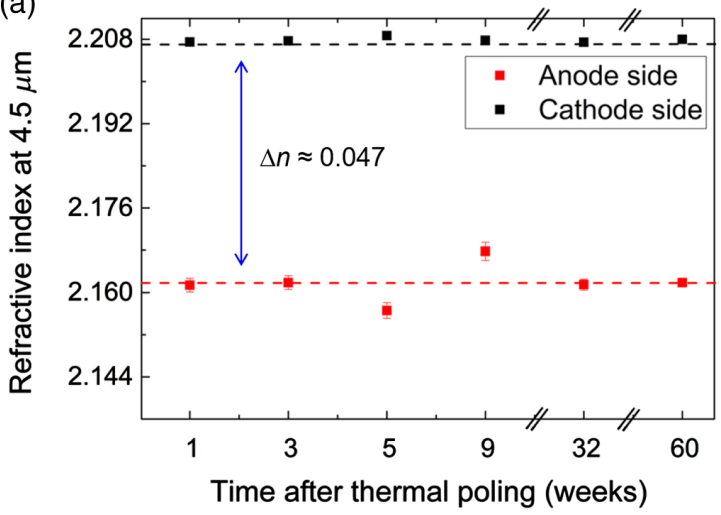

(b)

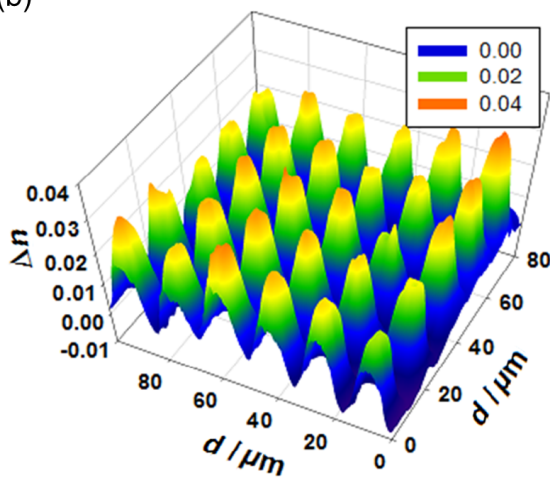

Fig. 3 (a) Postpoling refractive index stability measured over 60 weeks. The observed stability has now been documented beyond this period with no degradation to the magnitude of the compositional and refractive index change $(\Delta n)$ or the material's surface quality. (b) Qualitative measurements of the $\Delta n$ profile of an MLA, using phase imaging (Phasics SID4BIO) on B3-P glass. Target dimensions of the MLA's pitch were $\sim 17 \mu \mathrm{m}$. (a) reproduced with permission from Ref. 39. Copyright 2018 Springer Nature.

understanding those temperature as well as time-dependent conditions that dictate local nucleation and growth rates of the desired crystalline phases (dictating both the number density, crystallite size, and resulting filling fraction), phase fraction of both the induced crystalline phase and the (now depleted) residual glassy phase can be varied as a function of spatial position in a continuous manner. This control thus allows one to spatially vary the effective properties created by the multiphase media. Recent efforts by UCF researchers and their collaborators have employed this approach to realize optical composite media with GRIN profiles as well as spatially varying optical dispersion. This GRIN concept has been inspired by a wide range of phenomena in nature. For example, spatially varying refractive index profiles in human eyes enable imaging with good spatial resolution and low spectral aberration at both short and long distances. The lessons learned from nature can now be used to steer light in curved trajectories through structures such as a flat lens, which is also called a Wood lens as he pioneered the concept. ${ }^{74}$ The opportunities a GRIN flat lens (as an example) offers to enable a reduction in element size, weight, and component count, as well as manufacturing-related cost, thereby extending the trade 
space for optical performance parameters and increasing the design space needed for optical elements and resulting systems.

Among the most critical challenges for designing and fabricating a nanocomposite GRIN component is finding a material and a refractive index modification process that satisfies demanding manufacturing requirements with resulting index profiles that can be assessed with available metrology tools. These tools must not only quantify the resulting optical property gradients, but also the morphological/microstructural attributes of the materials that define the property modification over spatial dimensions down to a few hundred nanometers (for the IR). Typical material optical property requirements include spatial control of the sign, magnitude, and resolution of the refractive index of the candidate phase(s) in the composite and optical transparency of the element over a defined wavelength range. Similarly, the multiphase nature of the composite will impact the thermal and mechanical stability of the component as well as its compatibility with cost-effective manufacturing processes. These attributes will impact the resulting component's function and in-service performance stability. The candidate material must possess low optical loss in the spectral window of use and possess the ability to realize a suitably large refractive index change up to $\sim 0.1$ or dispersion modification combined with the ability to spatially modulate that index modification in an arbitrary manner in either the lateral and/or axial direction through the bulk optical component. Most importantly, the resulting modification must have postprocessed stability within defined operating conditions and be optically and physically compatible with other non-GRIN materials.

Recently, optical nanocomposites based upon multicomponent GAP-Se glasses have been shown to yield high-refractive index $\mathrm{Pb}$-containing nanocrystalline phases in an amorphous matrix, which can remain stable at elevated temperatures or under photo/electronically excited states. ${ }^{14,40-54}$ The effective refractive index, $n_{\text {eff }}$, of the resulting glass ceramic nanocomposite increases monotonically with an increase in the volume fraction of the high-refractive index crystalline phase(s), thereby enabling creation of a homogeneous effective refractive index change in the starting glass. The glass ceramic composite's effective index can be approximated by knowledge of the refractive index of the residual amorphous and induced crystal phase(s) formed as well as their respective volume fractions, as calculated by

$$
n_{\text {effective }} \approx V_{\text {matrix }} \times n_{\text {matrix }}+\sum_{i=1}^{N}\left(V_{i_{\mathrm{th}} \text { secondary phase }} \times n_{i_{\mathrm{th}} \text { secondary phase }}\right),
$$

where $n$ and $V$ correspond to the refractive index and volume fraction of the composite's phases, respectively. ${ }^{42,45,51,54}$ While simplistic and approximate, this relationship has been shown to reasonably estimate the effective optical properties observed in optical nanocomposites made up of glass and crystalline phase(s) in the glass ceramics formed from parent glass to spatially transform a medium to realize a new optical function. It is important to note that the remaining residual glass phase will be modified by the departure of the crystal-forming constituents, thus resulting in a modified postprocessed glassy phase refractive index, and due to the subtle nature of this depletion, this composition and index change are difficult to experimentally quantify. Within a host glass material system that can result in the formation of a secondary crystalline phase with a refractive index greater than that of the parent glass and low absorption in a spectral band of interest, spatial variation in the number density of such crystalline phases yields a gradient in refractive index and creates a desired low-loss transparent GRIN medium, as shown in Fig. 4(a). Such control over the desired low-loss crystalline phase requires knowledge of the crystal phase's nucleation rate $(I)$ and growth rate $(U)$ behavior, as shown in Fig. 4(b) for a glass with $20 \mathrm{~mol}$. \% PbSe. Knowledge of each glass' $I-U$ behavior, discussed in detail for small experimental melts ${ }^{43}$ and as a function of melt size, ${ }^{14}$ is critical to selecting an optimal time and temperature to create the desired crystalline phase.

Recent efforts have demonstrated the ability to engineer the index and dispersion behavior in bulk and thin film forms ${ }^{62}$ of GAP-Se materials with precision, including the ability to not only use laser-induced nucleation and/or heat-induced post growth to spatially control the localized crystal phase formation, ${ }^{14,40-54}$ but also the ability to locally induce vitrification (or reamorphization) of previously formed glass ceramics, to create a decrease in refractive index by converting previously formed nanocrystals back toward the initial, glassy state. ${ }^{54,55}$ Such 
(a)

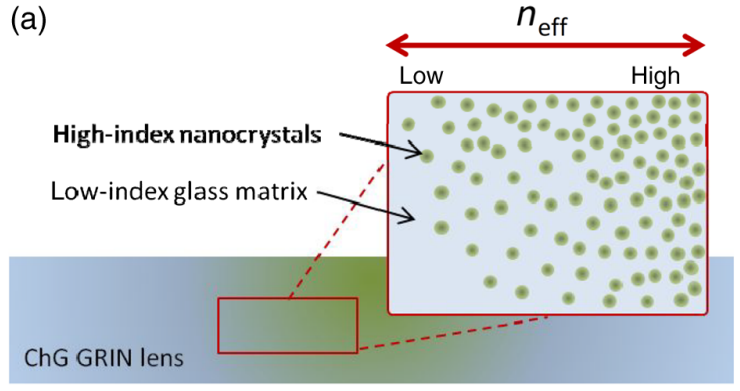

(b)

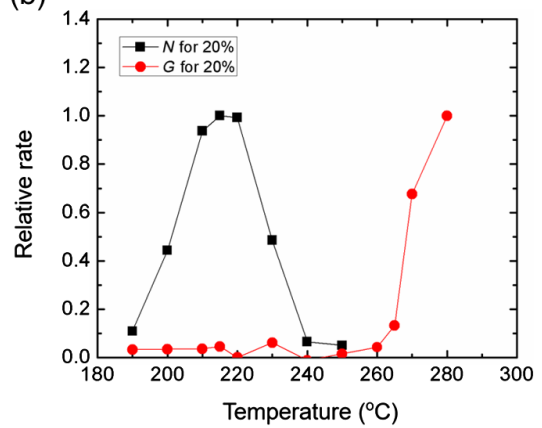

Fig. 4 (a) ChG GRIN lens where a gradient index is created through the spatial control of high refractive index particles embedded in a lower refractive index glass matrix. (b) The nucleation $(N)$ and growth-like $(G)$ rates for GAP-Se glasses with 20 mol. \% PbSe. (a) Reproduced with permission from Ref. 42. Copyright 2016 SPIE. (b) Reproduced with permission from Ref. 46. Copyright 2019 Wiley.

flexibility in both compositional design and processing routes will enable a wide variety of materials, which can be tailored to fit applications across the IR.

Bulk GRIN materials. Recently, Richardson's research group at UCF in collaboration with teams at Penn State and Lockheed Martin have found that GAP-Se glasses with PbSe content of $\sim 10$ to 45 mol. \% in their bulk form are spontaneously separated into Pb-rich and Pb-deficient amorphous phases upon standard melt-quench protocol. ${ }^{14,40-43,45,46,49,51-55}$ The energetically favorable route to phase separation consistently seen in this material system has gained interest from optical designers and glass scientists since the $\mathrm{Pb}$-rich amorphous phases, once thermally treated, can be exclusively converted into nanocrystallites with an index far greater than that of the surrounding matrix. . $^{14,41-43,45,46,49,51,53,54}$ This indicates that the effective index of nanocomposites increases upon heat treatment, while maintaining their transparency as an effective medium. Since the number density and the crystallinity of the high-refractive index, $\mathrm{Pb}$-rich phases can be controlled by knowledge of the nucleation and growth rates of these phases, thus a prescribed thermal treatment protocol, a combination of the material chemistry and the process, provides a pathway toward the formation of a GRIN profile within a single component. This has been experimentally validated through the use of a gradient heat (growth step) treatment to create a varying number of densities of $\mathrm{Pb}$-rich phases where the extent of their crystallinity, size, and volume fraction within a glass matrix enables one to spatially tune the effective index of the nanocomposite.

Specifically, varying the high index ingredient (in this case, the $\mathrm{Pb}$ content) in the parent glass leads to a modification in the base material's index and dispersion behavior. Figure 5(a) shows the GAP-Se ternary phase diagram where nine compositions of interest are represented by green dots. ${ }^{46}$ This variation in starting glass composition defines what possible crystal phases can be realized following heat treatment. Figure 5(b) shows the possible crystal phases that can form across the $\mathrm{Pb}$-series, following a single, unoptimized heat treatment schedule. ${ }^{46}$ As is shown in the legend, the key crystalline phases that form (the volume fractions of which are defined by the precise time and temperatures used in the heat treatment step) can include $\mathrm{PbSe}, \mathrm{GePbSe}$, $\mathrm{As}_{2} \mathrm{Se}_{3}$, and Se phases. Figure 5(c) shows the volume fractions of induced crystalline phases as a function of the parent glass's $\mathrm{PbSe}$ content where the volume fraction of the $\mathrm{Pb}$-containing high-index phases including $\mathrm{Ge}_{0.1} \mathrm{~Pb}_{0.9} \mathrm{Se}$ and $\mathrm{PbSe}$ monotonically increases with $\mathrm{PbSe}$ content, indicating that an increase in the effective refractive index of the nanocomposite upon heat treatment will likely occur with high index species forming at the expense of the parent glass species. ${ }^{45}$ As predicted by the effective index equation, the constituents in the glass are converted with heat treatment to form these crystal phases, thus depleting the glass (and changing its refractive index and volume fraction in the composite). This formation of crystal phases in the glass matrix gives rise to a modification in the material's refractive index and dispersion, as shown in 


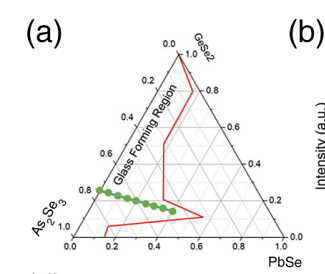

(b)

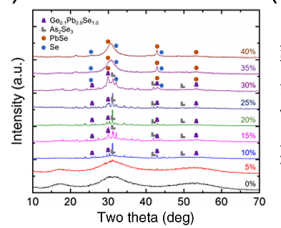

(c)

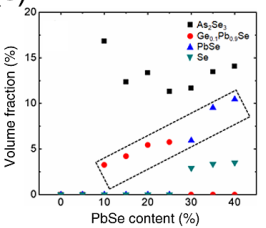

(f)

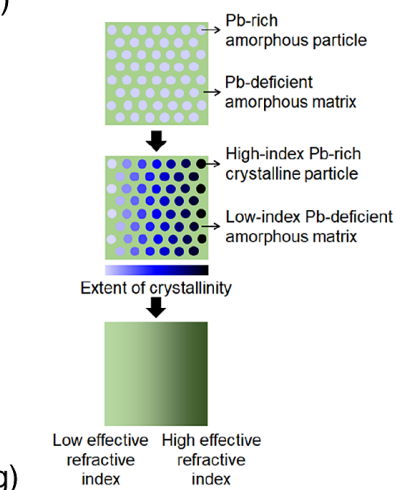

\section{(g)}

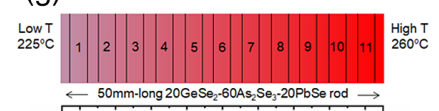

(e)

(d) 0 mol. \% PbSe $10 \mathrm{~mol} . \% \mathrm{PbSe} \quad 20 \mathrm{~mol} . \% \mathrm{PbSe} 30 \mathrm{~mol} . \% \mathrm{PbSe} 40 \mathrm{~mol}$ \% PbSe
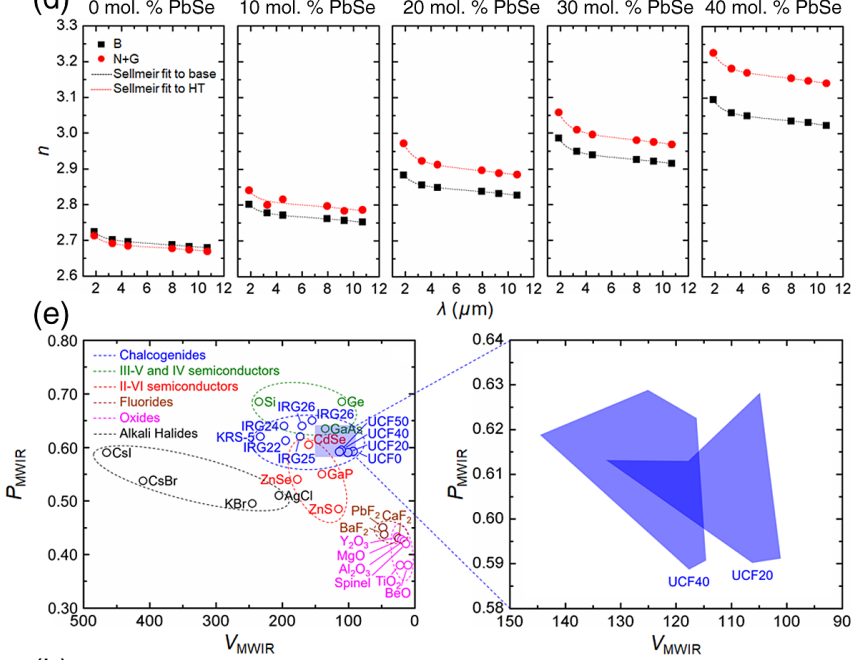

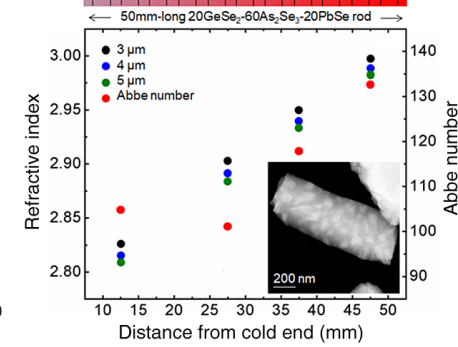

(h)

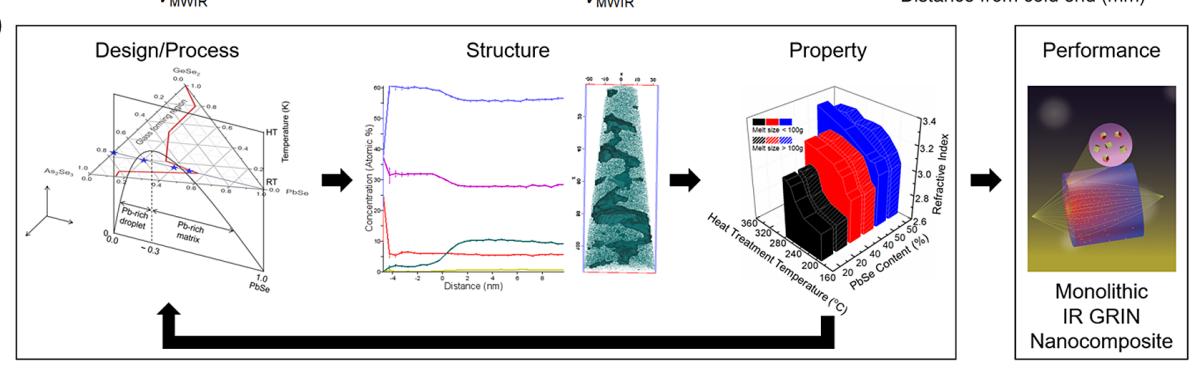

Fig. 5 (a) A room temperature ternary phase diagram of GAP-Se bulk material made upon meltquench protocol with compositions of interest, represented by green dots; (b) XRD spectra of heattreated samples with $\mathrm{PbSe}$ content ranging from 5 to $40 \mathrm{~mol}$. \%. Crystal phase identified from the peaks are indicated by the symbols, corresponding to $\mathrm{Ge}_{0.1} \mathrm{~Pb}_{0.9} \mathrm{Se}_{1.0}, \mathrm{As}_{2} \mathrm{Se}_{3}, \mathrm{PbSe}$, and $\mathrm{Se}$. (c) The volume fraction of the four crystalline phases crystalline observed in specimens with varying mol. \% of $\mathrm{PbSe}$ following the heat treatment. (d) Composition-dependent refractive index dispersion for base $(B)$ and nucleated and grown $(N+G)$ samples, prior to and following nucleation and growth. (e) (left) A refractive index dispersion map for various optical materials. (Right) The tailorability of MWIR dispersive properties for two different representative compositions of a GAP-Se system, enabled by heat treatment protocols. (f) Schematic illustrating microstructural evolution upon quenching and following gradient thermal treatment with a resulting GRIN profile created within the glass ceramic composite. (g) Gradient heat treatment of a 50-mm-long rod specimen with $20 \mathrm{~mol}$. \% PbSe in a gradient furnace. Measured refractive indices at $\lambda=3,4$, and $5 \mu \mathrm{m}$ and Abbe numbers based on the three MWIR wavelengths along with a corresponding TEM image of the specimen. (h) (left) Cyclic flowchart toward the development of monolithic IR GRIN nanocomposites where a specimen made based on a phase diagram-driven composition design and process protocol is structurally characterized to identify (middle) its morphology and chemistry in a 3-D space, (right) followed by the measurement of resulting optical properties such as refractive index modification, which is quantitatively correlated to material input parameters including composition and posttreatment, thereby providing feedback to the design/process step. (a), (b), and (d) Reproduced with permission from Ref. 46. Copyright 2019 Wiley. (c), (e), (f), (g), and (h) Reproduced with permission from Ref. 45. Copyright 2020 Wiley. 
Fig. 5(d) ${ }^{46}$ To illustrate the extent of dispersion engineering enabled by the glass ceramic formation process, Fig. 5(e) shows a refractive index dispersion map for groups of optical and IR materials including ChGs, III-V and IV semiconductors, II-VI semiconductors, fluorides, oxides, and alkali halides. ${ }^{45}$ Most material systems shown in the map have a single $V_{\text {MWIR }}-P_{\text {MWIR }}$ signature, indicating that the materials are limited to narrow ranges of dispersion. Unlike these homogeneous optical materials, the dispersive nature of the GAP-Se system can be tuned by thermally processing it to a glass-ceramic nanocomposite. The polygons in the magnified refractive index dispersion map correspond to the ranges of $V_{\text {MWIR }}-P_{\text {MWIR }}$ values enabled by current heat treatment protocols applied to two exemplary compositions including $20 \mathrm{~mol} \%$ and $40 \mathrm{~mol} . \% \mathrm{PbSe}$. These ranges quantitatively depict the tunability of dispersive properties for GAP-Se upon heat treatment, enabling options for overcoming the limitation that for most IR applications there are far fewer total materials available for use than those for visible systems.

Based upon experimental know-how of thermal protocol needed to achieve a specific refractive index modification, a prototype monolithic GRIN nanocomposite has been demonstrated. Here, the as-quenched glass with $20 \mathrm{~mol} \% \mathrm{PbSe}$, comprised of a Pb-rich amorphous secondary phase within a $\mathrm{Pb}$-deficient amorphous matrix, forms the starting morphology. A gradient furnace heat treatment is then applied to the glass where the extent of crystallization in the $\mathrm{Pb}$-rich secondary phases is tuned and increased by the spatially varying temperature profile within the furnace. As shown in Fig. 5(f), the spatially varying extent of crystallization correspondingly increases the effective refractive index of a resulting glass-ceramic material along the direction of the thermal gradient. ${ }^{45}$ Figure $5(\mathrm{~g})$ shows a schematic of the gradient thermal treatment applied to a 50-mm-long rod of GAP-Se glass with $20 \mathrm{~mol} \% \mathrm{PbSe} .{ }^{45}$ Here, the temperature extremes of the rod spans temperatures of $225^{\circ} \mathrm{C}$ near the furnace's cold end and $260^{\circ} \mathrm{C}$ near its hot end. The resulting glass-ceramic rod upon gradient heat treatment was cut into slices for subsequent characterizations, as represented by the numbers. As the extent of crystal formation (conversion) increases, one might expect this to impact the resulting optical composite's dispersive properties. The refractive indices and their MWIR Abbe numbers extracted from the pieces of the rod are shown in Fig. 5(g), where both of the values spatially vary. ${ }^{45}$ Notably, this specific heat treatment protocol and the straightforward conversion of a parent glass with this composition induces a refractive index and MWIR Abbe number gradients of $\sim 0.2$ and $\sim 35$, respectively. It is also noteworthy to mention that GAP-Se bulk glasses with $20 \mathrm{~mol} . \%$ of PbSe used in this study are known to develop multiple crystalline phases during heat treatment and temperatures at which each phase is nucleated are estimated to be different. The emergence of each crystalline phase is likely to induce a slight modification to the molecular bonds of its surrounding region in a different fashion, leading to the corresponding variation of Abbe number. The rod subjected to a gradient heat treatment has a spatially varying temperature profile along its longitudinal direction where the type of crystalline phases and the extent of corresponding modification to the surrounding region's molecular bonds are likely to be different at each location, leading to such a variation in Abbe number in the plot.

Meanwhile, primarily due to the GAP-Se glasses' irradiation-sensitive nature and the limitations of two-dimensional (2-D) morphology and microstructural assessment via traditional electron microscopic tools [i.e., transmission electron microscopy (TEM)], the true "as-melted" glass morphology and postheat treatment microstructure of this promising optical material has remained a lingering open question. The ability to know the precise compositional variation and by extension, volumetric phase, and index profile is essential to being able to predict the effective index profiles associated with a measured GRIN profile. Recently, atom probe tomography (APT) has been used to ascertain in three dimensions the ambiguous results provided by TEM cross-section observations. APT confirms the true connectivity of the crystalline phases developed upon heat treatment and provides a quantitative aspect in terms of the constituent atoms' spatial distribution. The complimentary APT-TEM study is expected to provide direct measurement of size, shape, composition, crystal types, and volume fractions, to enable a further refinement of inputs needed to the design-process-structure-property relationship of the optical nanocomposite system. Details of the APT method as applied to GAP-Se bulk materials are shown in Fig. 5(h). ${ }^{49,53}$ 
Planar GRIN materials. While bulk materials provide solutions that enable size, weight, and power (SWaP) reduction over homogeneous crystalline IR materials, GRIN films or lower dimensional structures formed from the GAP-Se material system also present an attractive alternative when used as conformal coatings on lower cost spherical surfaces. Here, aspheric function can be realized if film quality and GRIN profiles can be optimized through spatial control of the high index nanocrystalline phase. Demonstrated here, this approach exploits the fact that using thermal evaporation, GAP-Se films do not undergo phase separation upon their deposition. As the thermal history in the deposition is more energetic, the structure of deposited films remains far from equilibrium and thus, homogeneous and amorphous. ${ }^{44}$ However, the metastable films are energetically unstable; therefore, they are sensitive to external stimulation such as laser exposure and thermal treatment. ${ }^{44,47,48,50}$ This phenomenon can be utilized to create a GRIN profile within GAP-Se films high-Pb content [as represented by the red dot in Fig. 6(a)] where laser beam going through a grayscale mask induces spatially varying microstructural modification [Fig. 6(b)]. ${ }^{44,48}$ Specifically, Richardson's group has reported that laser exposure with a wavelength near the band edge of the film induces phase separation of the otherwise homogeneous amorphous structure into $\mathrm{Pb}$-rich amorphous phases within a $\mathrm{Pb}$-deficient amorphous matrix.

(a)

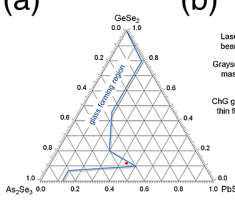

(c)

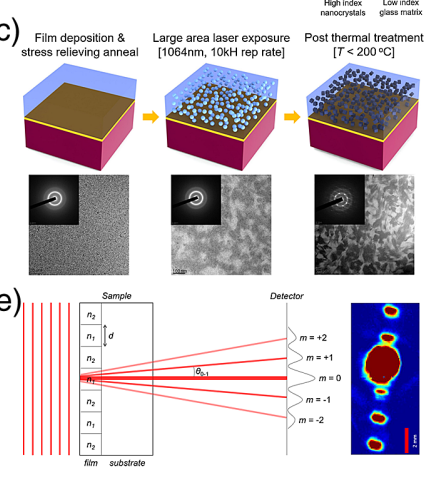

(d)

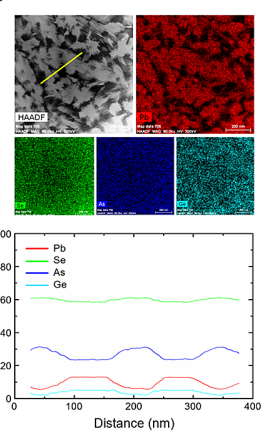

(g)

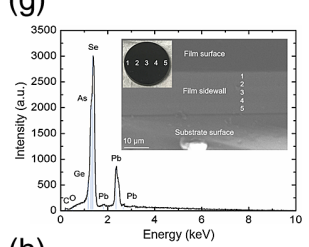

(h)
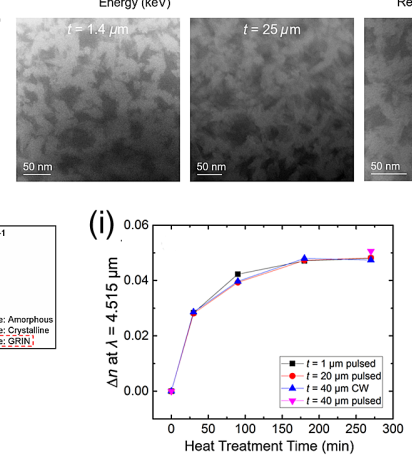

Fig. 6 (a) The phase diagram of GAP-Se glass produced via a melt-quench protocol with the composition of interest represented by the red dot. The concentration of nanocrystals is spatially modulated by varying the exposure dose across the film through the use of a grayscale mask, producing a highly controlled index gradient along the lateral direction. (b) Fabrication process flow for the controlled nanocrystal growth in a GAP-Se film and corresponding TEM images + selected area electron diffraction (SAED) patterns showing microstructural evolution at each step of the photothermal process. (c) The diffractive performance of the laser-written and postannealed grating structure measured in transmission at $\lambda=2 \mu \mathrm{m}$ where multiple diffraction spots are clearly observed, confirming an index modulation within the structure. (d) Composition analysis of GAPSe films following the photothermal process where the TEM image, XEDS maps, and spatial linear composition profiles of the constituents indicate the spatial segregation of $\mathrm{Pb}$ atoms. (e) Abbe numbers for common IR media and GAP-Se glasses across SWIR, MWIR, and LWIR spectral bands. (f) (left) Photo/SEM image of 1-in. wide, 25- $\mu$ m-thick GAP-Se film, along with EDS data from multiple locations throughout film's volume. (Right) Homogeneous atomic percentages of constituents for lateral positions and axial positions. (g) Cross-sectional TEM images of films with thicknesses of $1.4,20$, and $40 \mu \mathrm{m}$ where coexisting $\mathrm{Pb}$-rich, high-index, crystalline phases (bright), and a Pb-deficient, low-index, amorphous phase (dark) can be shown in each image, as well as the consistency of their size and distribution between images. (h) The change in refractive index measured at different heat treatment times at $\lambda=4.515 \mu \mathrm{m}$ for varying film thicknesses following photothermal process. (b)-(e) Reproduced with permission from Ref. 44. Copyright 2018 Wiley. (g) Reproduced with permission from Ref. 48. Copyright 2019 SPIE. (h) and (i) Reproduced with permission from Ref. 47. Copyright 2020 Elsevier. 
Since the laser exposure-induced $\mathrm{Pb}$-rich amorphous phase is unstable against crystallization, postheat treatment leads to the formation of high refractive index $\mathrm{Pb}$-rich nanocrystals within a low-refractive index Pb-deficient matrix, as summarized in Fig. 6(c). ${ }^{44}$ Figure 6(d) shows X-ray energy dispersive spectroscopy (XEDS) maps and corresponding linear composition profiles of constituent atoms collected from a resulting glass-ceramic film where the spatial segregation of $\mathrm{Pb}$ is evident. ${ }^{44}$ Laser exposure can be locally modulated, and it has been shown that it is total fluence that dictates the number density of $\mathrm{Pb}$-rich amorphous phases, which become high refractive index phases upon postheat treatment. The effective refractive index, $n_{\text {eff }}$, of the resulting glass-ceramic nanocomposite films increases with the volume fraction of the high refractive index crystalline phase(s) and has been utilized to realize a GRIN component such as a diffraction grating structure, as shown in Fig. 6(e). ${ }^{44}$ While the homogeneous dispersive properties of the GAPSe film were found to be similar to other conventional broadband materials, the radial GRIN Abbe number was found to be orders of magnitude greater across the full bandwidth, indicating the ultralow dispersion of refractive index, thereby making this material an ideal candidate for spectral aberration correction [Fig. 6(f)] ${ }^{44}$ Meanwhile, the optical path difference (OPD) is one of key metrics that quantifies the performance of an optical medium as a GRIN lens. The quantity is formulated by the $\Delta n \times t$ product where $\Delta n$ and $t$ correspond to a medium's refractive index change and thickness along its optical path, respectively. With a given GRIN profile within a medium, the OPD increases with the medium's thickness. This has led to the demonstration of the ability to laterally modulate effective refractive index change up to $\sim 0.1$ within GAP-Se films, in films with thicknesses up to $40 \mu \mathrm{m} .{ }^{48}$ Specifically, reported here is the demonstration of optical functionality within films possessing a wide range of thicknesses from 1 to $40 \mu \mathrm{m}$, confirming that the films are compositionally homogeneous [Fig. 6(g)], ${ }^{48}$ and the photothermal process is both repeatable and scalable, as the thickness of the film does not factor into microstructural and index modifications [Figs. 6(h) and 6(i)]. ${ }^{47,50}$

\subsubsection{GRIN via spatially controlled vitrification}

While thermal-only or photothermal processes are promising as strategies create GRIN optics in parent glasses, an additional strategy has been developed whereby a spatially varying profile can be realized in a material that exhibits a transition between glass and glass-ceramic states. Richardson's group has utilized an approach reverse to the laser-assisted crystallization technique. ${ }^{54,55}$ Specifically, the strategy, termed "laser-induced vitrification (LIV)," involves starting with a glass-ceramic and inducing a change in effective refractive index by locally reversing the extent of crystallization by melting of the existing nanocrystals, as shown in Fig. 7(a). ${ }^{55}$ A lasermodified region possesses a higher volume fraction of glassy phase with a lower index than the surrounding glass-ceramic material. The LIV technique based on absorption-induced heating was developed for GAP-Se bulk glasses where a subsurface grating, as a form of arrays of vertical pillars induced by translational movement of laser irradiation, was fabricated [Fig. 7(b)]. ${ }^{55}$ The white light interferometry (WLI) data of the LIV-written grating indicate excellent uniformity with a grating period close to that designed by the laser-written pillar patterns, as shown in Fig. 7(c). ${ }^{55}$ Figure 7(d) shows that the technique has been successfully used to create and demonstrate, for the first time, clear optical functionality in the form of an IR diffraction grating. ${ }^{55}$ Specifically, the LIV-written grating structure with a grating periodicity of $60 \mu \mathrm{m}$ in Fig. 7(c) was characterized in both transmission $(\lambda=2 \mu \mathrm{m})$ [Fig. 7(d) top] and reflective $(\lambda=0.632 \mu \mathrm{m})$ [Fig. 7(d) bottom] modes to quantify its diffractive properties. The spacings between the zeroorder and first-order spots in the reflection and transmission modes correspond to reflection and transmission diffraction angles of $2.90 \mathrm{deg}$ and $2.76 \mathrm{deg}$, respectively. A refractive index difference between a region above a pillar and an interpillar region within the near-surface volume grating is estimated to be $\Delta n \sim-0.056$ based on the grating equation. The predicted value closely matches that $(-0.062)$ extracted from the Raman-converted index map in the study with an error of $12.5 \%,{ }^{55}$ confirming that LIV-induced local decrease in refractive index is evident and consistent with what the grating equation implies. The LIV-induced microstructural and optical modifications, which result in such a diffraction grating performance, have been evidenced through a variety of characterization methods including TEM, micro $(\mu)$-XRD, and Raman spectroscopy. First, Fig. 7(e) shows cross-sectional TEM images collected underneath the LIV-written grating where 
(a)

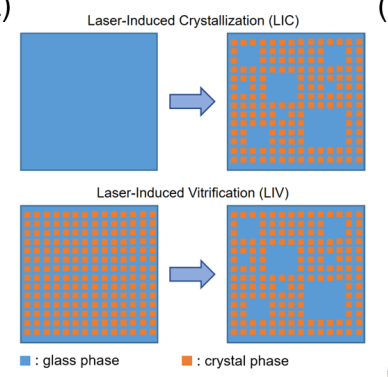

(b)

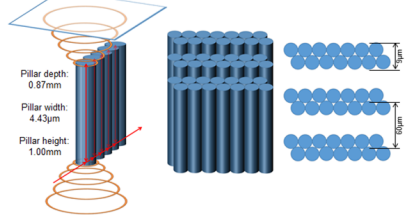

(c)

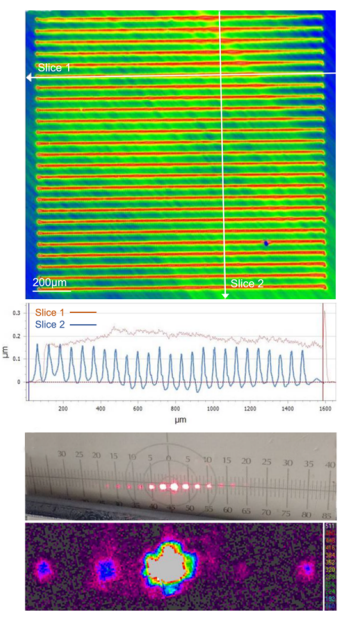

(e)

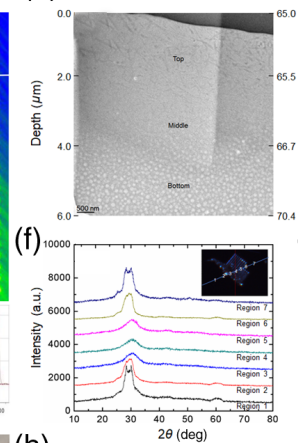

(h)

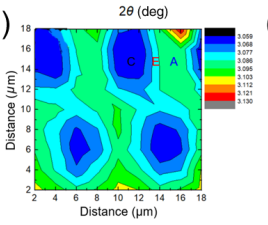

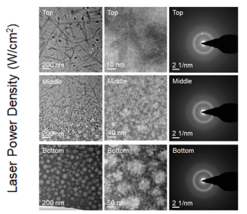

(g)

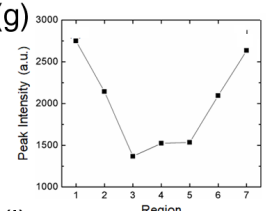

(i)

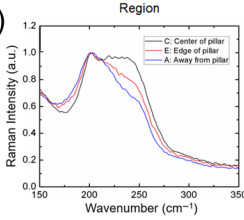

Fig. 7 (a) Comparison of laser-assisted modification strategies including laser-induced crystallization (LIC) and LIV. (Top) LIC employs a homogeneous glass material (blue) and selectively grows crystal phases (red) in specific locations. (Bottom) LIV, the reverse of LIC, reamorphizes local regions through spatially selective laser exposure and vitrification. (b) Laser irradiation geometry with its translational direction along red arrows and resulting arrays of pillars. The concentric rings are meant to depict the varying focal area associated with the beam waist above and below the focal point. (c) A WLI image of the laser-written grating surface illustrating the line patterns resulting from the pillar exposure modification to the bulk glass ceramic. Surface relief measured along a single laser written line and across all grating lines. (d) Diffractive properties of the laserwritten grating measured in (top) reflection at $\lambda=0.632 \mu \mathrm{m}$ and (bottom) transmission at $\lambda=2 \mu \mathrm{m}$. (e) (left) TEM images overlaid with a depth-dependent laser power density profile and corresponding high-magnification TEM images + SAED patterns for the top, middle, and bottom regions of the cross-sectional TEM specimen. (f) Grazing-incidence $\mu$-XRD spectra collected from laser-induced grating regions and regions away from the structure. (g) A plot of integrated crystal phase peak intensity extracted from the XRD spectra for the positions identified in (f). (h) A Ramanconverted refractive index profile map of a grating strip with four pillars that make up the strip. The legend indicates the refractive index scale corresponding to the colors shown. C (black), E (red), and $\mathrm{A}$ (blue) represent positions in the region above the center of a pillar, at the edge of a pillar, and in an unirradiated region away from a pillar. (i) The corresponding Raman spectra collected at the three locations in (h). (a)-(i) reproduced with permission from Ref. 57. Copyright 2018 OSA.

there is a depth-dependent microstructural transition from near-surface glass-ceramic to fully revitrified structures, which has been shown to be consistent with the computed laser power density as a function of depth. ${ }^{55}$ Postprocessed regions were shown to exhibit microscopic features suggestive of phase-separation similar to that observed in the parent glass prior to glass ceramic formation. Second, $\mu$-XRD was performed on multiple locations in and away from the laser-induced grating to assess the spatial variation of crystallinity, as shown in Figs. 7(f). ${ }^{55}$ To quantify the crystallinity of each region, the intensity of the dominant peak located at $2 \theta \approx 30 \mathrm{deg}$, originated from $\mathrm{PbSe}$ and $\mathrm{As}_{2} \mathrm{Se}_{3}$ crystalline phases, is extracted from each spectrum and is plotted in Fig. 7(g), where the peak intensity, proportional to the crystallinity of a region of interest, decreases in the laserirradiated pattern, while the value increases in the region away from the grating. ${ }^{55}$ The Raman map directly correlated to refractive index modification and spectra collected above, at the edge of, and away from a pillar collectively suggest that the extent of LIV is quantified to be $62 \%$, and postprocessing refractive index modification is realized to be -0.062 [Figs. 7(h) and 7(i)].

\subsection{Additional Strategies for Planar GRIN Materials}

\subsubsection{Three-dimensional printing of solution-derived ChG glasses}

Spatial control and variation of the physical properties in IR transparent glasses is a desirable attribute that not only is useful in bulk optics, but also in thin films that are compatible with other 
(a)

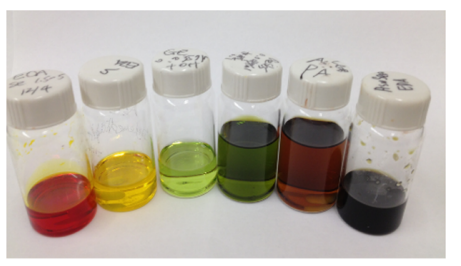

(b)

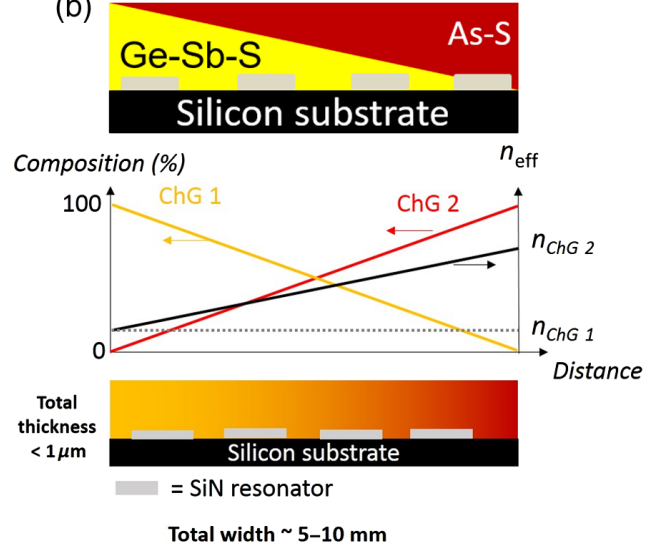

Fig. 8 (a) Multiple solutions of various ChG materials ${ }^{88}$ dissolved in amine solvents. (b) (top) Bilayer design of a GeSbS and AsS gradient profile and (bottom) actual effective index profile of the measured gradient layer, as a function of position across a SiN resonator array. (b) Reproduced with permission from Ref. 56. Copyright 2017 ACS Publications.

on-chip materials and components. Efforts by numerous groups over the past decade have shown that ChGs are soluble in a variety of amine-based solvents. ${ }^{75}$ First demonstrated by teams led by coauthors Richardson ${ }^{76-78}$ and $\mathrm{Hu}^{79,80}$ with Craig Arnold at Princeton, ${ }^{79,81-83}$ it was shown that solution-derived $\mathrm{ChG}$ films could be processed with physical properties closely matching that of the parent bulk material. These efforts, largely focused on multimaterial, chip-scale ( $\mathrm{Si}$ ) integration of ChGs for sensing applications, and formed the basis of further activities aimed at doping with nanoparticles or quantum dots whereby luminescent behavior could extend the optical functionality of the films. ${ }^{84,85}$ To aid in the physical dispersion of such particles to create tailorable optical function without quenching, coating techniques were further optimized to develop an electrospray (ES) technique ${ }^{86,87}$ whereby particles could be contained within aerosol droplets, which upon evaporation of solvent during deposition, particles would not undergo the typical agglomeration realized in spin coating and other coating techniques. Once optimized, ES was shown to be suitable for depositing multiple composition of $\mathrm{ChG}$ and enabled the systematic creation of multilayer films. By altering the thickness of each film, with knowledge of the ES spot film profile and resulting postheat-treated film thickness, effective index gradient layers were created. ${ }^{44}$ These direct print structures can be tailored for thickness, index, and thus the resulting effective index profile defined by the path of the three dimensional (3-D) printing profile. Multiple solutions of various $\mathrm{ChG}$ compositions following dispersion in amine solvents is shown in Fig. 8(a) ${ }^{88}$ Figure 8(b) shows the gradient film deposition profile of a bilayer of GeSbS and AsS glasses (on $\mathrm{Si}$ ), and the variation in effective index, $n_{\text {eff }}$, calculated based on the layer thickness and parent glass refractive indices, as deposited on a SiN resonator array. ${ }^{56}$ Such deposition requires an electrically conductive substrate (i.e., $\mathrm{Si}$ ), but is amenable to plano and other shaped substrates. In addition, the magnitude of the resulting index gradient is only defined by the film thickness and the excursion $(\Delta n)$ between the candidate glasses used.

\subsubsection{Photo-induced polymerization of ChG glasses}

Since the first efforts to write optical functionality into oxide glasses with lasers, glasses have been evaluated for their use as waveguides, ${ }^{89}$ 2-D waveguide arrays, ${ }^{90}$ resonators, and other refractive or diffractive optical elements. ${ }^{91}$ Such structures have been realized by laser-writing, usually using femtosecond laser pulses or by lithographic processing of glassy layers deposited by a range of film deposition techniques. Planar thin films based on ChGs have been long known to be photosensitive, as evidenced by their use in xerographic processes ${ }^{92}$ and as resists for lithographic applications. ${ }^{93}$ This photosensitivity has been found to arise from defect "tail" states associated with normal and abnormal (over or undercoordinated electron- or hole-containing) bonds within the chalcogen-containing amorphous semiconductor network. Extension of laserwriting approaches to nonoxide materials specifically to write on-chip optical structures and 


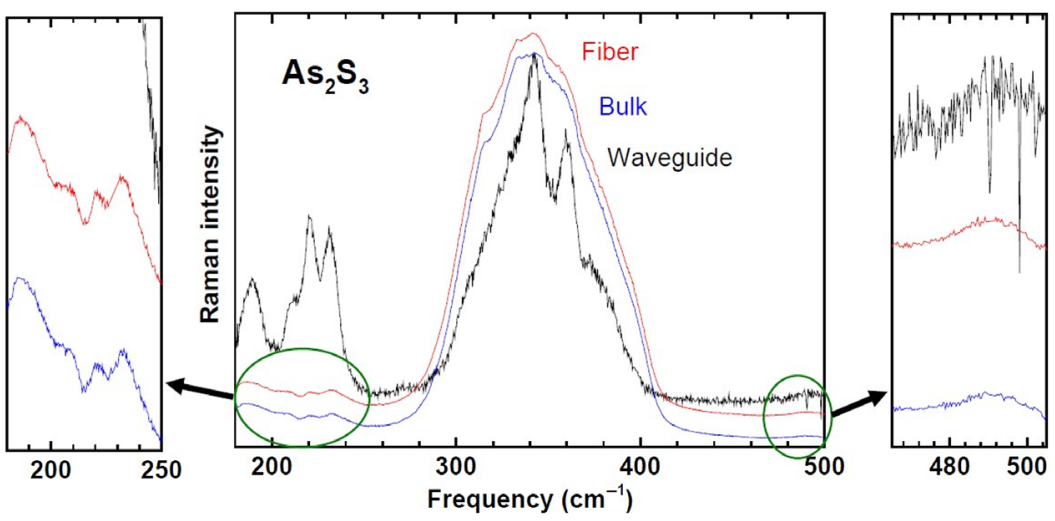

Fig. $9 \mu$-Raman spectra illustrating heteropoloar (As-S) and homopolar bonds (As-As and S-S) in $\mathrm{As}_{2} \mathrm{~S}_{3}\left(\lambda_{\text {exc }}=840 \mathrm{~nm}\right)$ measured with $1.5 \mathrm{~cm}^{-1}$ spectral resolution. Highlighted in the circled areas are the higher concentration of homopolar (As-As and S-S) bonds found in films/waveguides (features in the 200 to 250 and $490 \mathrm{~cm}^{-1}$ region, respectively) as compared to bulk and fiber forms of the material. Theoretically, stoichiometric material should only possess heteropolar (As-S) bonds. These abnormal, homopolar bonds not present to the same extent in the bulk and fiber forms and render films much more photosensitive. Reproduced with permission from Ref. 98. Copyright 2002 Wiley.

to understand the fundamental differences in properties between bulk and thin films ${ }^{94,95}$ and the mechanisms of photostructural modification ${ }^{96}$ was initiated by the Richardson UCF team in conjunction with collaborators at Laval University, in the mid- to late 1990s. Figure 9 shows the variation in as-formed structural units within glasses of $\mathrm{As}_{2} \mathrm{~S}_{3}$ in bulk, thin film, and fiber form, highlighting the presence of "abnormal" bonds in ChG films, as quantified by Raman spectroscopy. ${ }^{97,98}$ Note especially the presence of homopolar As-As and S-S bonds, which impart higher photosensitivity in as-deposited films or waveguides, as compared to bulk glass.

Using the device fabrication and optical characterization tools from the Laval team in conjunction with the glass processing and characterization tools at UCF, quantification for the first time showed variations in optical and physical properties as a result of forming processes and extension of bulk glasses to film form. As discussed in the literature, most as-deposited ChGs (bulk glass targets or crystalline alloy targets are typically amorphous following film deposition $^{99}$ ) exhibit photosensitivity, specifically the ability to change their optical properties (band gap or refractive index) through the thermal or photonic modification of localized bonds. Modification of such materials with varying types of irradiation can lead to the creation of unique optical functionality, including enhanced nonlinearities. The photosensitivity of ChGs in laserwritten structures has been well studied (see the review contained in Ref. 100), and the optical function and stability of gratings waveguides have been shown to be a function of glass's composition, the wavelength of light used to write the structure, and the environment where photoformation aging takes place. ${ }^{101-107}$

Most studied by the UCF researchers ${ }^{108,109}$ and others is $\mathrm{As}_{2} \mathrm{~S}_{3}$, whereby planar films can be doped or locally altered to realize gratings and waveguides. While responsive, these as-written structures and their optical property stability are sometimes short-lived, in that light-induced bond-reorganization may lead to localized crystallization or postexposed modification, which anneals out at room temperature due to the glass's low glass transition temperature $\left(T_{\mathrm{g}}\right)$. Recent efforts led by the Kuebler team at $\mathrm{UCF}^{59}$ have exploited the ease of glass network reorganization imparted by photoinduced bond modification, to realize structures which exhibit etch selectivity between bonds contained in the modified region as compared to unirradiated material. This processing has been employed to realize 2-D and 3-D structures with a wide range of optical functionality. Defined as multiphoton lithography (MPL), systematic dose-dependent material modification studies have been carried out to realize metastructures.

Figure 10(a) shows a 2-D array of the $\mathrm{As}_{2} \mathrm{~S}_{3}$ pillars over a $250 \times 250 \mu \mathrm{m}^{2}$ region realized using direct laser writing (DLW) with a wavelength of $800 \mathrm{~nm} .{ }^{59}$ Similar irradiation conditions were used to realize gratings that could be tuned [Figs. 10(b)-10(f)] to realize diffractive features 


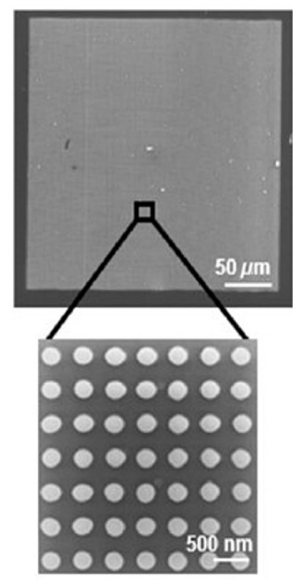

(a)

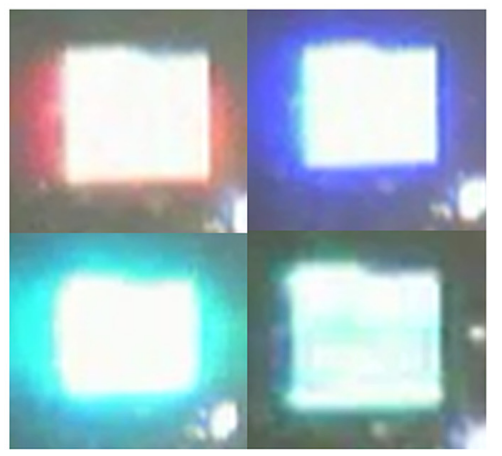

(e)

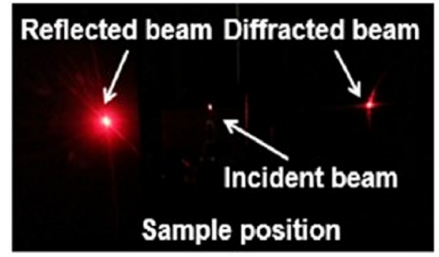

(b)

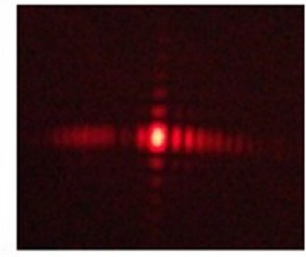

(d)

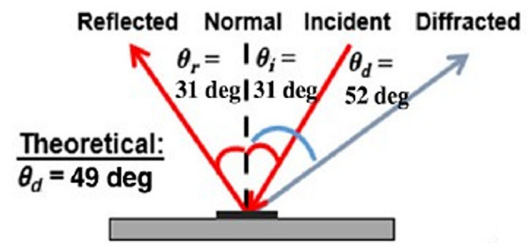

(c)

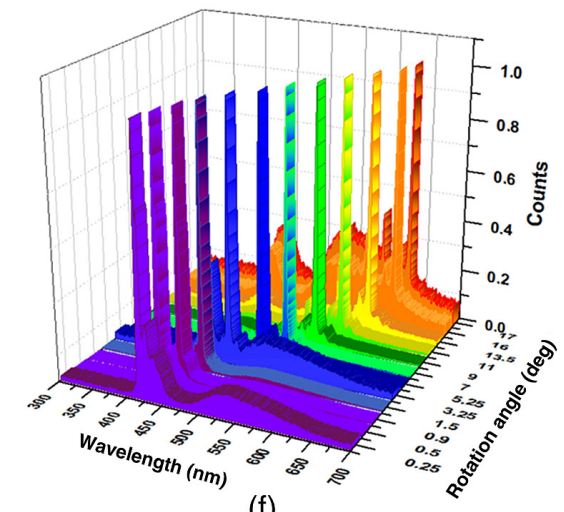

(f)

Fig. 10 (a) SEM image of the $250 \times 250 \mu \mathrm{m}^{2}$ nanostructured pillar array $\left(\mathrm{As}_{2} \mathrm{~S}_{3}\right)$ with a close-up image showing the tops of the individual structures. (b) Optical image of the incident, reflected, and diffracted beams. (c) Diagram depicting the actual angle for the reflected and diffracted beams and the predicted angle for the diffracted beam. (d) Close up optical image of the actual refracted beam pattern. (e) Optical images of a $250 \times 250 \mu \mathrm{m}^{2}$ nanostructured pillar array showing that the structure diffracts light of different colors as the angle of incidence is changed. (f) Plot of the diffracted light intensity versus wavelength of white light incident at varying angles. (a) Reproduced with permission from Ref. 59. (b)-(f) Reproduced with permission from Ref. 57. Copyright 2017 SPIE.

that exhibited spectral-specific response. ${ }^{57}$ These structures exhibit good optical functionality, but suffer from limited stability due to the crystallization tendency of these ChGs films if not stabilized after writing/etching. As part of a currently funded DARPA program, ${ }^{110}$ the UCF team with our colleagues at LMCO and MIT has extended this previously demonstrated capability to more complex, thicker structures $(t \sim 4 \mu \mathrm{m})$ such as patterned arrays and Fresnel structures on both flat and curved (planoconvex ZnSe lens) surfaces. Such structures made using the same laser wavelength are shown in Fig. 11. While not specific to the GRIN process, such DLW plus optimized etch protocols offer unique opportunities of writing conformal coatings with added optical function on glass or crystalline optical surfaces.

While the spatial control and periodic uniformity in etch behavior exhibited in the large area grid shown in Fig. 11(b) is excellent and the DLW of $\mathrm{As}_{2} \mathrm{~S}_{3}$ glass films has been shown to be a versatile process that exploits the photosensitivity of this glass system, the long-term stability of this glass is not optimal. Hence, modification of the glass structure with an additional constituent, Ge, known to enhance glass network cross-linking, has been shown to enhance the long-term stability of the film. This addition reduces the film's photoresponse; however, the film still exhibits etch selectivity (albeit, at a level reduced as compared to the binary As-S glass films). More importantly, the Ge addition dramatically improves the post write/etch stability, demonstrating film-aging stability for periods (measured) up to 4 years. ${ }^{111}$ A postaged array of a GeAsSe 
(a) Process flow

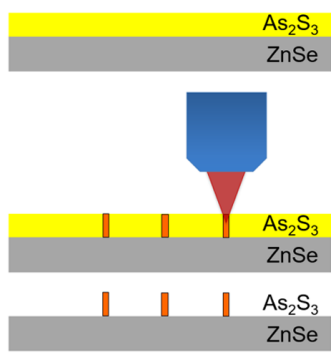

(b) Planar platform

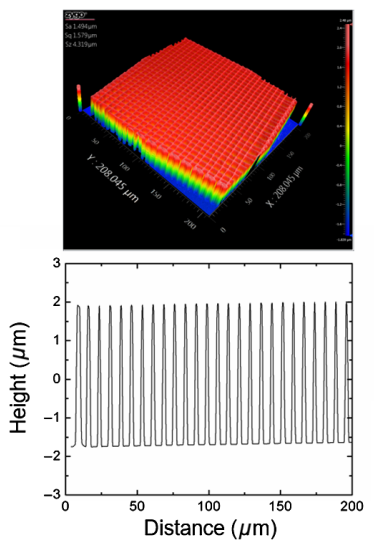

(c) Curved platform
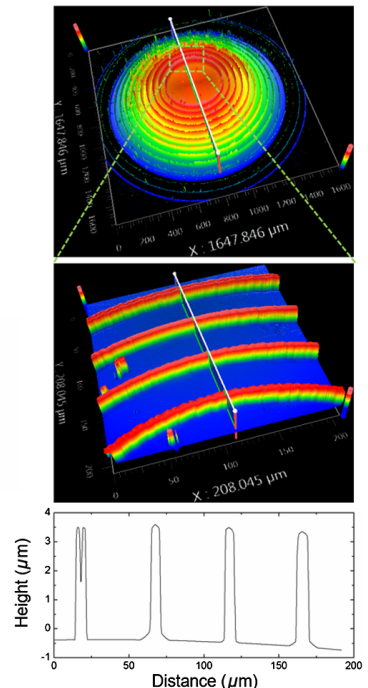

Fig. 11 (a) A schematic of two-step photochemical process flow where large area, laser-written metasurface structures are realized via selective chemical etching. (b) A demonstration of a large area, high fidelity grid structures (7.5- $\mu \mathrm{m}$ grid spacing) on a planar platform. (c) A demonstration of laser written and etched circular ring patterns on a curved IR lens platform.
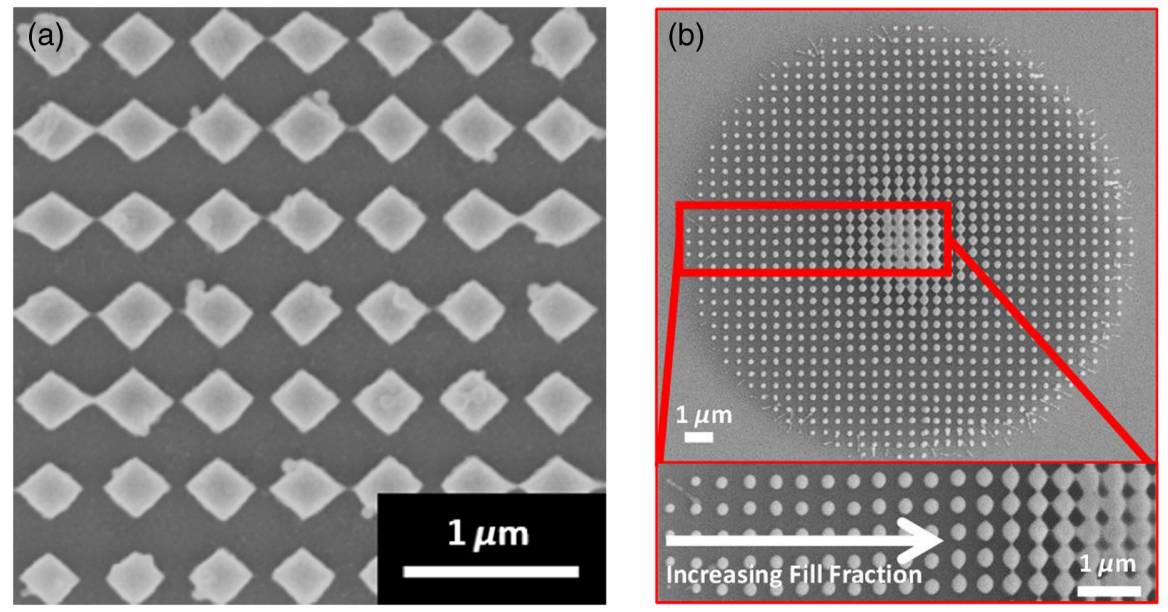

Fig. 12 (a) Ge-containing As-Se film array pattern $\left[\mathrm{Ge}_{5}\left(\mathrm{As}_{40} \mathrm{Se}_{60}\right)_{95}\right]$ after $t=4$ years of aging. Features show minor deterioration at their edges but retain their as-written lateral dimensions.

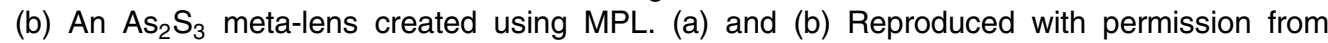
Ref. 111. Copyright 2018 OSA.

structure is shown after 4 years in Fig. 12(a). By tailoring the composition (for stability) and filling fraction (of glass pillar material and free volume of air) of an area where such a pattern exists [Fig. 12(b)], a gradient effective index (here in the form of a metalens structure) can be realized.

\subsection{Optical Design and Metrology Tools for GRIN Materials and Components}

As methods to develop new GRIN materials for the IR continue to evolve, so too do efforts to model and design GRIN media to predict their optical performance. In parallel, advances to establish robust IR characterization methodologies to quantify GRIN media through viable optical metrology methods have also seen progress within the past decade. Discussed briefly here are 
select recent advances on methods suitable to IR materials that have extended the state-of-the-art over the past decade.

\subsubsection{Optical design tools for GRIN}

As part of the DARPA M-GRIN program initiated in the 2010 timeframe, an investment in the development and testing of optical design tools that could model and predictively assess strategies that could lead to reduction in SWaP requirements for optical systems was undertaken. This work, initiated largely on visible materials, was extended to the IR as two M-GRIN teams were focused on IR media as their target platform. Hence, tools were needed that could expand beyond tools available with commercial design codes, such as CODE V and ZEMAX. ${ }^{12,113}$ While multiple groups were involved in these efforts, the GRIN-specific results came from efforts led by the Werner team at Penn State University. The tools realized by this group were transferred to a commercial startup in 2014, named E $\times \mathrm{H}$, Inc., which carried out numerous test studies of various tools. The group's ReTort tool was initially developed to support efforts in transformation optics and design strategies to reduce $\mathrm{SWaP}^{114,115}$ and has since been extended to treat other types of applications including metamaterials. Early work showed how methods could be used for color and aberration correction design ${ }^{116,117}$ along with multifrequency wavefront matching. ${ }^{118}$ In Ref. 118, the team blended material dispersion attributes afforded by GRIN mixtures and metasurfaces to realize large diameter apochromatic components. Though not exhaustive, these works (and the references therein) have led to the realization of key design tools applicable to GRIN coatings and bulk component design across the visible and IR.

IR systems deployed in extreme environments such as space or military regions require stable optical performance over an extended operating temperature range. The ever-growing usage of IR systems in such conditions has necessitated an optical design method that accurately takes into account the thermo-optic coefficient of optical glasses and its desirable adoption to inhomogeneous material combinations. Recent work by Boyd at Qioptiq has shown noteworthy progress on this approach where a model for the first-order optical design of athermal, radial GRIN systems is utilized. ${ }^{119,120}$ Specifically, the work has suggested that radial GRIN components can significantly reduce the optical power balance of athermal, achromatic systems, thereby reducing aberration associated with individual lens components and improving overall performance. The improved first-order modeling technique provides a new opportunity for the optimization of a broadband multispectral optical design. As an example, SWIR/long wavelength infrared (LWIR) optical design for a weapon-sight application has been shown to have significantly reduced mass and improved performance compared with a conventional non-GRIN solution. ${ }^{119,120}$

\subsubsection{Optical metrology tools for GRIN}

Historically, GRIN metrology methods have been similar to those techniques employed for other optical characterization of traditional homogeneous bulk optical components based on interferometry. Interferometric approaches for evaluating homogeneous visible optical media have been the preferred approach to quantify the impact of glass melting and annealing protocols on resulting refractive index homogeneity. ${ }^{121}$ Commercial sources for such testing now exist to quantify these attributes in both the visible and IR. ${ }^{122}$ While it is desirable in homogeneous optical components that the within part index uniformity is high [typically $10^{-4}$ to $10^{-6}$ refractive index units or better), defects in the processing methodology of the media can result in localized imperfections or more spatially global errors that result in wavefront transmission degradation. Such defects arise from density variations associated with incomplete/inadequate mixing or poor annealing, thus resulting in striae. These local defects are visible in interferograms, and therefore, are the go-to method for visually mapping imperfection that can limit optical performance. These tools have recently been applied together with experimental ray-tracing methods for 3-D-printed optics to assess resulting optical quality of prototype bulk GRIN components made from multicomposition glass "feedstock." 123

Such interferometric tools, when extended to GRIN materials, often do not span the appropriate spectral range or can be destructive in nature. Methods employing Raman mapping ${ }^{124}$ and 
Richardson et al.: Advances in infrared gradient refractive index (GRIN) materials: a review

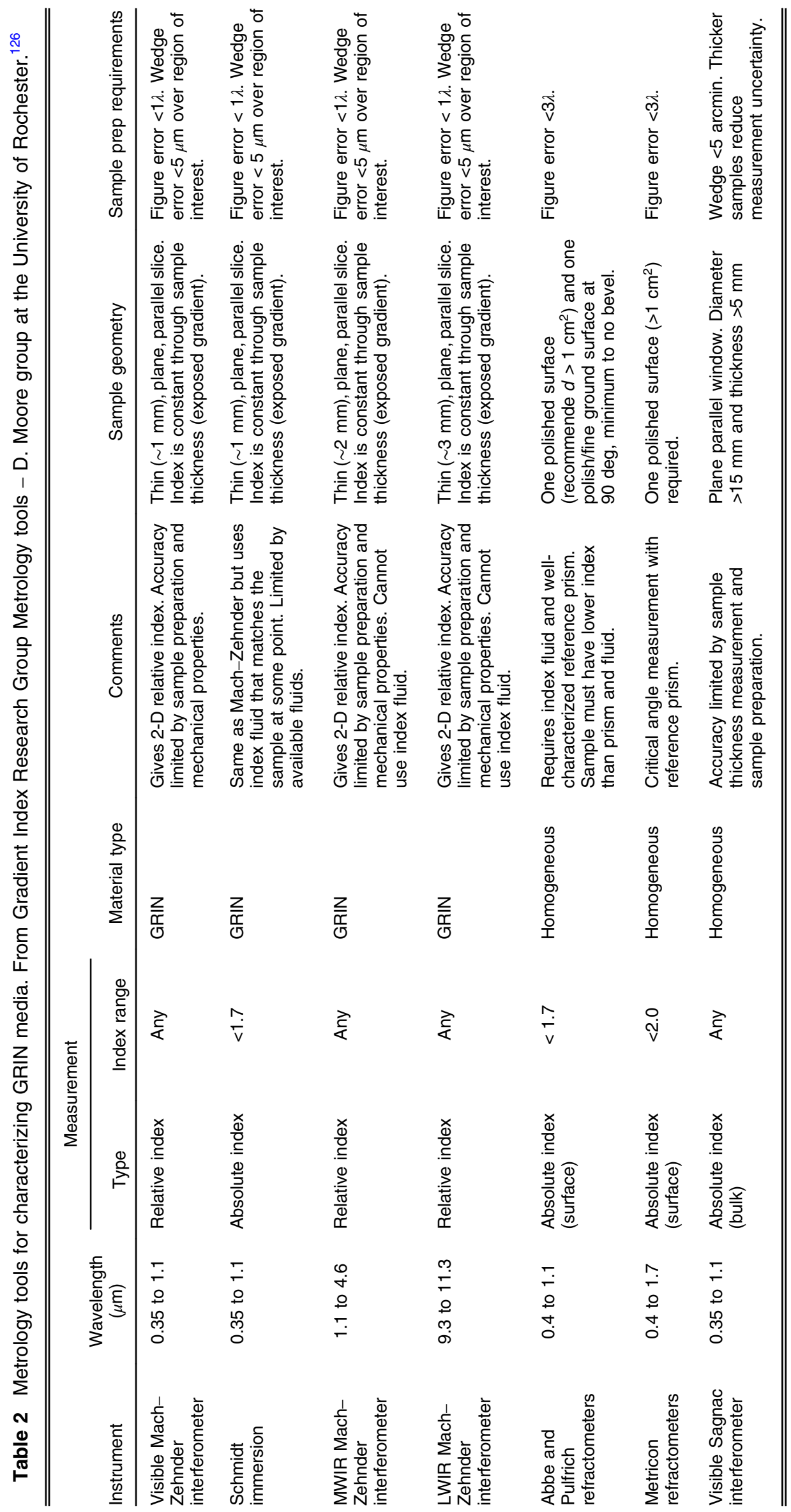




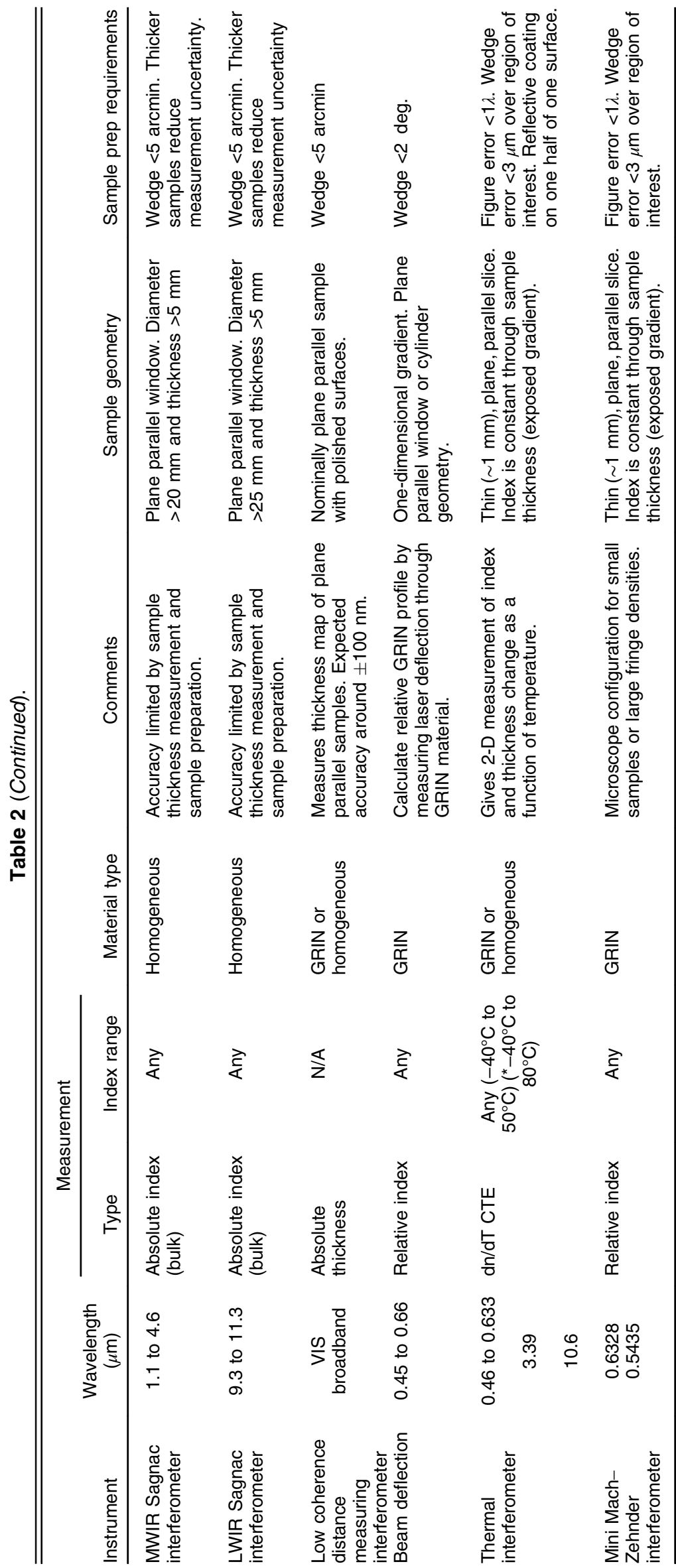


optical coherent tomography ${ }^{125}$ have been applied to both polymers and glasses with some success. These tools have recently been expanded into the IR to allow use on IR GRIN materials with mixed results. Here, these tools work well where sharp variations in indices are seen, such as in layered media. However, they become less accurate when there is no defined spatial variation, such as in media which have been interdiffused or have dispersed nanoscale crystallites. As with the interferometric characterization for homogeneous optical components, the key attributes of GRIN media that are important to quantify via GRIN metrology methods include within-part refractive index uniformity or step profile (either continuous or stepwise) in both the radial and axial directions. The Gradient Index Research Group at the University of Rochester (UofR) led by Prof. Duncan Moore has led in the study of GRIN media for more than 40 years. Appropriately, some of the most comprehensive instrumentation for characterizing both homogeneous and GRIN media throughout the visible and IR reside at UofR. Tables 1-3 summarize these tools and their attributes as well as the limitations of the methods. ${ }^{126}$ In addition to the limitations mentioned in the tables, it is also important to note that tools do not often possess the spatial resolution to enable accurate quantification of steep gradients or arbitrary gradient profiles. Specifically, for a refractive index spatially varying within an area smaller than the minimum beam spot size provided by an instrument, which is typically a few millimeters, a refractive index averaged out over the area is measured rather than its spatially varying profile. To overcome the issue, the Richardson group has demonstrated an alternative technique where a spectral map provided by a micro-Raman is correlated to the spatial distribution of refractive index modification at a micron-scale, as shown in Figs. 7(h) and 7(i). ${ }^{54,55}$ A comprehensive evaluation of strategies for the design and measurement of GRIN in the IR can be found in Ref. 127. Specific examples employing design and measurement strategies are described in Refs. 60, 128, and 129. While not exhaustive, these publications highlight many of the key issues when considering the use of IR materials. Specific examples applied to optical components and systems are discussed in Refs. 130-134.

Table 3 Typical error and limitations of various metrology tools. From the Gradient Index Research Group Metrology tools - D. Moore group at the University of Rochester. ${ }^{126}$

\begin{tabular}{|c|c|c|c|}
\hline Instrument & $\begin{array}{l}\text { Best case } \\
\text { error bar }\end{array}$ & Advantages & Disadvantages \\
\hline $\begin{array}{l}\text { Mach-Zehnder } \\
\text { interferometer }\end{array}$ & $2 \times 10^{-5}$ & $\begin{array}{l}\text {-Very versatile and robust } \\
\text { measurement method } \\
\text {-Schmidt immersion method } \\
\text { provides absolute index }\end{array}$ & $\begin{array}{l}\text {-Sample prep problems can limit } \\
\text { measurement accuracy } \\
\text {-Sample prep is typically destructive }\end{array}$ \\
\hline $\begin{array}{l}\text { Abbe and } \\
\text { Pulfrich } \\
\text { refractometers }\end{array}$ & $1 \times 10^{-5}$ & -Robust measurement method & $\begin{array}{l}\text {-Limited by reference prism and } \\
\text { available index fluids } \\
\text {-Surface measurement only }\end{array}$ \\
\hline $\begin{array}{l}\text { Sagnac } \\
\text { interferometer }\end{array}$ & $1 \times 10^{-4}$ & $\begin{array}{l}\text {-Average index measurement } \\
\text {-Used for any index or wavelength } \\
\text { range } \\
\text {-Uses plane parallel sample rather } \\
\text { than prism }\end{array}$ & $\begin{array}{l}\text {-Sensitive to scatter and surface } \\
\text { shape }\end{array}$ \\
\hline Beam deflection & $1 \times 10^{-3}$ & $\begin{array}{l}\text {-Nondestructive test for certain } \\
\text { geometries } \\
\text {-Can be implemented in-process }\end{array}$ & $\begin{array}{l}\text {-Requires constant index in } \\
\text { propagating direction }\end{array}$ \\
\hline $\begin{array}{l}\text { Thermal } \\
\text { interferometer }\end{array}$ & $\begin{array}{l}\text { CTE and } \\
d n / d T \text { to two } \\
\text { or three digits }\end{array}$ & $\begin{array}{l}\text {-Gives CTE and } d n / d T \text { as a } \\
\text { function of }(x, y) \\
\text {-Useful for GRIN and homogeneous } \\
\text { materials }\end{array}$ & $\begin{array}{l}\text {-Double pass (reduces effective } \\
\text { fringe resolution) } \\
\text {-Requires environmentally } \\
\text { controlled chamber }\end{array}$ \\
\hline
\end{tabular}




\section{Conclusions}

The recent history of efforts aimed at realizing GRIN across a range of IR-transparent materials is reviewed. The ability to exploit material composition, photosensitivity, processing methodology, and resulting optical function, specifically highlighting the trade-offs and scale-ability of solutions, has been presented. Multiple examples of strategies in bulk and thin film ChGs are discussed along with progress made in the development of optical design tools and metrology methods in the IR. Specifically, metrology tools, which more readily assess the performance of materials, optical components, and systems, continue to be developed. The recent efforts by the IR community to evaluate alternate strategies to add optical functionality to elements suitable for use in this important region of the spectrum highlight the promising future of such approaches to reduce the SWaP requirements needed for next-generation optical components and systems.

\section{Acknowledgments}

The authors acknowledge numerous sources of support associated with this work. KR acknowledges the contributions of former team members associated with prior results, and specifically, Dr. Benn Gleason who contributed extensively to the preparation of GeAsSe bulk and thin film glasses and their optical characterization to assess structural stability. We also note the collaborative partnership and partial financial support for the MPL work led by Professor Steve Kuebler (SK), with funding provided through NSF CAREER award DMR/CHE-0748712 and DMR1337758. We (SK and KR) acknowledge funding from Lockheed Martin, the Florida High-Tech Corridor Council, the Space Research Initiative Program through the Florida Space Institute at the University of Central Florida, and the National Aeronautics and Space Administration (NASA) through UCF's NASA Florida Space Grant Consortium. We acknowledge the helpful contributions of Anna Lewis, Gerald Richardson, and Shreya Labh who were supported by UCF SURF scholarships as part of efforts on MPL of ChGs. We also thank Professor Pieter Kik and Mr. Chatdanai Lumdee for assistance with ellipsometry measurements of postlaser written films and solution-derived films. KR and $\mathrm{SN}$ acknowledge the partnership of colleagues in the Microphotonics Laboratory and the Department of Materials Science and Engineering at MIT and the support of DTRA through contract HDTRA-13-1-0001. KR and Antoine Lepicard (AL) acknowledge the collaborative partnership of Dr. Marc Dussauze at the University of Bordeaux and partial funding support of the NSF, through award DMR-1308946, Programme IdEx Bordeaux - LAPHIA (ANR-10-IDEX-03-02), the French Aquitaine region (20121101025), and AST Microlens. The authors jointly acknowledge the characterization support of UCF's Material Characterization Facility, specifically Mr. Mikhail Klimov, for support on SIMS measurements and Michel Lahaye at PLACAMAT (UMS 3626) in Bordeaux, for the microprobe measurements on the $\mu$-poling results. Dr. Myungkoo Kang (MK) acknowledges financial support of UCF's Pre-eminent Postdoctoral Program (P3). KR also acknowledges the prior contributions in GRIN material/component design and development by multiple collaborators, including Dr. Charmayne Lonergan, Andrew Buff, Dr. Claudia Goncalves, Mia Truman, Jun Qin, Michael Antia, Justin Cook, Dr. Fedor Kompan, Dr. Chris Grabill, Dr. Johann M. Veras, Professor Ivan Divliansky, Professor Leonid B. Glebov, Ian B. Murray, Professor Martin C. Richardson, Professor Carlo G. Pantano, Dr. Alexej Pogrebnyakov, Andrew Kirk, Samantha Mensah, Megan Driggers, Dr. Anuradha Agarwal, Professor Juejun Hu, Professor Pao-Tai Lin, Dr. Cheng Li, and Professor Weiwei Deng, for various aspects of analyses and useful discussions related to the goals of this work. Finally, this work was supported in part by Defense Advanced Research Projects Agency (DARPA) under Air Force Research Laboratory contract FA8650-12-C-7225 through the M-GRIN Tech Area 2 program, and contract HR-00111720029 (DARPA-EXTREME program). The authors acknowledge the support of M-GRIN program collaborators at Penn State University and ExH, Inc., (Professor Doug Werner and Dr. Sawyer Campbell) for their input regarding GRIN-specific optical design methods, as well as partners at the University of Rochester (UofR), led by Professor Duncan Moore and Dr. Greg Schmidt. The UofR team's efforts to work with M-GRIN team members as they developed improved GRIN materials proved invaluable to the program and beyond. The views, opinions, and/or findings expressed 
are those of the authors and should not be interpreted as representing the official views or policies of the Department of Defense or the U.S. Government.

\section{References}

1. B. Gleason et al., "Refractive index and thermo-optic coefficients of GeAsSe chalcogenide glasses," Int. J. Appl. Glass Sci. 7, 374 (2016).

2. B. Gleason et al., "Designing mid-wave infrared (MWIR) thermo-optic coefficient (dn/dT) in chalcogenide glasses," Proc. SPIE 9822, 982207 (2016).

3. J. M. Jewell, C. Askins, and I. D. Aggarwal, "Interferometric method for concurrent measurement of thermo-optic and thermal expansion coefficients," Appl. Opt. 30, 3656 (1991).

4. P. O. McLaughlin and D. T. Moore, "Measurement of the differential thermal expansion and temperature dependence of refractive index in gradient-index glass," Appl. Opt. 24, 4334 (1985).

5. P. O. McLaughlin and D. T. Moore, "Models for the thermal expansion coefficient and temperature coefficient of the refractive index in gradient-index glass," Appl. Opt. 24, 4342 (1985).

6. P. McCarthy et al., "Application of a multiple cavity Fabry-Perot interferometer for measuring the thermal expansion and temperature dependence of refractive index in new gradient-index materials," in Imaging and Appl. Opt. (1986).

7. B. Ananthasayanam et al., "Final shape of precision molded optics: Part II validation and sensitivity to material properties and process parameters," J. Thermal Stresses 35, 614 (2012).

8. G. Guery et al., "Evolution of glass properties during a substitution of $\mathrm{S}$ by $\mathrm{Se}$ in $\mathrm{Ge}_{28} \mathrm{Sb}_{12} \mathrm{~S}_{60-\mathrm{x}} \mathrm{Se}_{\mathrm{x}}$ glass network," J. Non-Cryst. Solids 358, 1740 (2012).

9. B. Ananthasayanam et al., "Final shape of precision molded optics: Part I computational approach, material definition and the effect of lens shape," J. Thermal Stresses 35, 550 (2012).

10. S. Gaylord et al., "Thermal and structural property characterization of commercially moldable glasses," J. Am. Ceram. Soc. 93, 2207 (2010).

11. E. Koontz, "Characterization of structural relaxation in inorganic glasses using length dilatometry," PhD Dissertation, Clemson University (2014).

12. E. Koontz, P. Wachtel, and K. Richardson, "Compositional dependence of structural relaxation behavior in the Ge- As-Se system characterized by length dilatometry," in OSA Top. Meeting Opt. Fab. and Testing (OF\&T), Kona, Hawaii, p. OM2C.4 (2014).

13. C. R. Petersen et al., "Mid-infrared supercontinuum covering the 1.4-13.3 $\mu \mathrm{m}$ molecular fingerprint region using ultra-high NA chalcogenide step-index fibre," Nat. Photonics 8, 830 (2014).

14. A. Yadav et al., "Effect of melt size and scale-up protocols on property evolution in $\mathrm{GeSe}_{2}-\mathrm{As}_{2} \mathrm{Se}_{3}-\mathrm{PbSe}$ (GAP-Se) glass ceramics for infrared GRIN applications," Intl. J. Appl. Glass Sci. 10, 27 (2019).

15. K. Richardson, "Optimization of manufacturability of chalcogenide glasses for midinfrared optical components," in OSA Top. Meeting Opt. Fab. and Testing (OF\&T), Kona, Hawaii, p. OM2C.5 (2014).

16. J. Morris et al., "Achrotech: achromat cost versus performance for conventional, diffractive, and GRIN components," Proc. SPIE 9947, 994704 (2016).

17. S. D. Fantone, "Refractive index and spectral models for gradient-index materials," Appl. Opt. 22, 432 (1983).

18. M. A. Pickering, R. L. Taylor, and D. T. Moore, "Gradient infrared optical material prepared by a chemical vapor deposition process," Appl. Opt. 25, 3364 (1986).

19. S. Ohmi et al., "Gradient-index rod lens made by a double ion-exchange process," Appl. Opt. 27, 496 (1988).

20. J. R. Hensler, inventor; Bausch, Lomb Inc., assignee, "Method of producing a refractive index gradient in glass," US patent US 3,873,408 (1975).

21. S. Houde-Walter and D. T. Moore, "Real-time index profile measurement during GRIN glass fabrication," Appl. Opt. 27, 508 (1988). 
22. L. G. Atkinson et al., "Design of a gradient-index photographic objective," Appl. Opt. 21, 993 (1982).

23. G. Beadie et al., "Optical properties of a bio-inspired gradient refractive index polymer lens," Opt. Express 16, 11540 (2008).

24. R. A. Flynn and G. Beadie, "Athermal achromat lens enabled by polymer gradient index optics," Proc. SPIE 9822, 98220S (2016).

25. L. E. Busse et al., "Chalcogenide-glass clad fiber delivers high power at mid-IR wavelengths," (1995).

26. I. D. Aggarwal and J. S. Sanghera, "Development and applications of chalcogenide glass optical fibers at NRL," J. Optoelectron. Adv. Mater. 4, 665 (2002).

27. O. P. Kulkarni et al., "Third order cascaded Raman wavelength shifting in chalcogenide fibers and determination of Raman gain coefficient," Opt. Express 14, 7924 (2006).

28. J. S. Sanghera and I. D. Aggarwal, "Active and passive chalcogenide glass optical fibers for IR applications: a review," J. Non-Cryst. Solids 256, 6 (1999).

29. V. Kamensky et al., "High-power As-S glass fiber delivery instrument for pulse YAG:Er laser radiation," Appl. Opt. 37, 5596 (1998).

30. E. Papagiakoumou, D. N. Papadopoulos, and A. A. Serafetinides, "Pulsed infrared radiation transmission through chalcogenide glass fibers," Opt. Commun. 276, 80 (2007).

31. X. H. Zhang, H. Ma, and J. Lucas, "Evaluation of glass fibers from the Ga-Ge-Sb-Se system for infrared applications," Opt. Mater. 25, 85 (2004).

32. J. Teichman et al., Gradient Index Optics at DARPA, The Institute for Defense Analyses, Alexandria, Virginia (2014).

33. D. Gibson et al., "IR GRIN optics: design and fabrication," Proc. SPIE 10181, 101810B (2017).

34. D. Gibson et al., "IR-GRIN optics for imaging," Proc. SPIE 9822, 98220R (2016).

35. D. Gibson, S. Bayya, and J. Sanghera, "Homogeneous and gradient index (GRIN) materials for multi-band IR optics," in Classical Opt., Optical Society of America (2014).

36. D. Gibson et al., "Layered chalcogenide glass structures for IR lenses," Proc. SPIE 9070, 90702I (2014).

37. D. J. Gibson et al., "GRIN optics for multispectral infrared imaging," Proc. SPIE 9451, 94511P (2015).

38. A. Lepicard et al., "Dimensional control and stabilization of second harmonic electrooptical response in chalcogenide glasses," Opt. Mater. Express 8, 1613 (2018).

39. A. Lepicard et al., "Long-lived monolithic micro-optics for multispectral GRIN applications," Sci. Rep. 8, 7388 (2018).

40. A. Yadav et al., "Influence of phase-separation on structure-property relationships in the $\left(\mathrm{GeSe}_{2}-3 \mathrm{As}_{2} \mathrm{Se}_{3}\right)_{1-x} \mathrm{PbSe}_{x}$ glass system,” Phys. Chem. Glasses- $B$ 58, 115 (2017).

41. L. Sisken et al., "Evidence of spatially selective refractive index modification in a $15 \mathrm{GeSe}_{2}-45 \mathrm{As}_{2} \mathrm{Se}_{3}-40 \mathrm{PbSe}$ glass ceramic through correlation of structure and optical property measurements for GRIN applications," Opt. Mater. Express 7, 3077 (2017).

42. K. Richardson et al., "Engineering novel infrared glass ceramics for advanced optical solutions," Proc. SPIE 9822, 982205 (2016).

43. A. Buff, "The effects of phase separation on crystallization in $\mathrm{GeSe}_{2}-\mathrm{As}_{2} \mathrm{Se}_{3}-\mathrm{PbSe}$ glasses," Master's Thesis, University of Central Florida (2016).

44. M. Kang et al., "Ultra-low dispersion multicomponent thin film chalcogenide glass for broadband gradient index optics," Adv. Mater. 30, 1803628 (2018).

45. M. Kang et al., "Monolithic chalcogenide optical nanocomposites enable infrared system innovation: gradient refractive index (GRIN) optics," Adv. Opt. Mater. 8, 2000150 (2020).

46. L. Sisken et al., "Infrared glass ceramics with multi-dispersion and gradient refractive index attributes," Adv. Funct. Mater. 29, 1902217 (2019).

47. I. Mingareev et al., "Spatial tailoring of the refractive index in infrared glass-ceramic films enabled by direct laser writing," Opt. Laser Technol. 126, 106058 (2020).

48. M. Kang et al., "Scalable laser-written Ge-As-Pb-Se chalcogenide glass-ceramic films and the realization of infrared gradient refractive index elements," Proc. SPIE 10998, 109980E (2019). 
49. H. Francois-Saint-Cyr et al., "Three-dimensional microstructural characterization of novel chalcogenide nanocomposites for gradient refractive index applications," Microsc. Microanal. 25, 2500 (2019).

50. I. Mingareev et al., "Laser-induced modification of local refractive index in infrared glassceramic films," Proc. SPIE 10906, 109060X (2019).

51. K. Richardson et al., "Advances in infrared GRIN: a review of novel materials towards components and devices," Proc. SPIE 10627, 106270A (2018).

52. C. Goncalves et al., "New candidate multicomponent chalcogenide glasses for supercontinuum generation," Appl. Sci. 8, 2082 (2018).

53. M. Kang et al., "Unveiling true three-dimensional microstructural evolution in novel chalcogenide nanocomposites as a route to infrared gradient refractive index functionality," Microsc. Microanal., in press (2020).

54. L. Sisken, "Laser-induced crystallization mechanisms in chalcogenide glass materials for advanced optical functionality," PhD Dissertation, University of Central Florida (2017).

55. M. Kang et al., "Refractive index patterning of infrared glass ceramics through laserinduced vitrification," Opt. Mater. Express 8, 2722 (2018).

56. S. Novak et al., "Direct print of multilayer gradient refractive index chalcogenide glass coatings by electrospray," ACS Appl. Mater. Interfaces 9, 26990 (2017).

57. C. M. Schwarz et al., "Fabrication and characterization of micro-structures created in arsenic trisulfide chalcogenide glasses by multi-photon lithography," J. Micro/ Nanolithogr., MEMS, MOEMS 16, 023508 (2017).

58. C. M. Schwarz et al., "Multi-photon lithography of 3D microstructures in $\mathrm{As}_{2} \mathrm{~S}_{3}$ and $\mathrm{Ge}_{5}\left(\mathrm{As}_{2} \mathrm{Se}_{3}\right)_{95}$ chalcogenide glass," Proc. SPIE 9759, 975916 (2016).

59. C. M. Schwarz et al., "Fabrication and characterization of micro-structures created by direct laser writing in multi-layered chalcogenide," Proc. SPIE 9374, 937403 (2015).

60. A. J. Yee and D. T. Moore, "Free-space infrared Mach-Zehnder interferometer for relative index of refraction measurement of gradient index optics," Opt. Eng. 56, 111707 (2017).

61. J. Yao et al., "Nondestructive metrology by optical coherence tomography empowering manufacturing iterations of layered polymeric optical materials," Opt. Eng. 52, 112111 (2013).

62. R. Myers, N. Mukherjee, and S. R. Brueck, "Large second-order nonlinearity in poled fused silica," Opt. Lett. 16, 1732 (1991).

63. T. G. Alley, S. R. J. Brueck, and M. Wiedenbeck, "Secondary ion mass spectrometry study of space-charge formation in thermally poled fused silica," J. Appl. Phys. 86, 6634 (1999).

64. Y. Quiquempois et al., "Localization of the induced second-order non-linearity within Infrasil and Suprasil thermally poled glasses," Opt. Commun. 176, 479 (2000).

65. Y. Quiquempois et al., "Second-order nonlinear susceptibility in $\mathrm{As}_{2} \mathrm{~S}_{3}$ chalcogenide thin glass films," Electron. Lett. 36, 733 (2000).

66. J. Qiu, J. Si, and K. Hirao, "Photoinduced stable second-harmonic generation in chalcogenide glasses," Opt. Lett. 26, 914 (2001).

67. M. Dussauze et al., "Device and method for inducing by thermal poling a spatially controlled refractive index gradient inside an amorphous inorganic material," European Patent application \#EP16176689.4 (2016).

68. M. Dussauze et al., "Structural rearrangements and second-order optical response in the space charge layer of thermally poled sodium-niobium borophosphate glasses," J. Phys. Chem. C 111, 14560 (2007).

69. M. Dussauze et al., "Thermal poling of optical glasses: mechanisms and second-order optical properties," Int. J. Appl. Glass Sci. 3, 309 (2012).

70. M. Dussauze et al., "Accurate second harmonic generation microimprinting in glassy oxide materials," Adv. Opt. Mater. 4, 929 (2016).

71. A. Lepicard et al., "Micro-structuring the surface reactivity of a borosilicate glass via thermal poling," Chem. Phys. Letts. 664, 10 (2016).

72. A. Lepicard et al., "Surface reactivity control of borosilicate glasses using thermal poling," J. Phys. Chem. C 119, 22999 (2015).

73. A. Lepicard, "Design of surface chemical reactivity and optical properties in glasses," $\mathrm{PhD}$ Dissertation, University of Central Florida/University of Bordeaux (2016). 
74. R. W. Wood, Physical Optics, MacMillan, New York (1905).

75. K. Richardson et al., "Progress on the fabrication of on-chip, integrated chalcogenide glass (ChG)-based sensors," J. Nonlinear Opt. Phys. Materi. 19, 75 (2010).

76. N. Carlie, "A solution-based approach to the fabrication of novel chalcogenide glass materials and structures," PhD Dissertation, Clemson University (2010).

77. J. W. Novak, "Solution-derived chalcogenide glass films for MIR planar sensors," Master's Thesis, Clemson University/University of Bordeaux 1 (2012).

78. S. Novak, "Nanoparticles in solution-derived chalcogenide glass films," Master's Thesis, Clemson University/University of Bordeaux 1 (2012).

79. Y. Zou et al., "Solution processing and resist-free nanoimprint fabrication of thin film chalcogenide glass devices: inorganic-organic hybrid photonic integration," Adv. Opt. Mater. 2, 759 (2014).

80. Y. Zou et al., "High-performance, high-index-contrast chalcogenide glass photonics on silicon and unconventional non-planar substrates," Adv. Opt. Mater. 2, 478 (2014).

81. S. Song et al., "Spin-coating of $\mathrm{Ge}_{23} \mathrm{Sb}_{7} \mathrm{~S}_{70}$ chalcogenide glass thin films," J. Non-Cryst. Solids 355, 2272 (2009).

82. J. Hu et al., "Development of chipscale chalcogenide glass based infrared chemical sensors," Proc. SPIE 7945, 79452C (2011).

83. M. Waldmann et al., "Structural properties of solution processed $\mathrm{Ge}_{23} \mathrm{Sb}_{7} \mathrm{~S}_{70}$ glass materials," J. Mater. Chem. 22, 17848 (2012).

84. C. Li et al., "Electrospray deposition of quantum dot-doped $\mathrm{Ge}_{23} \mathrm{Sb}_{7} \mathrm{~S}_{70}$ chalcogenide glass films," Thin Solid Films 626, 194 (2017).

85. V. Singh et al., "Mid-infrared materials and devices on a Si for optical sensing," Sci. Technol. Adv. Mater. 15, 014603 (2014).

86. S. Novak et al., "Electrospray deposition of uniform thickness $\mathrm{Ge}_{23} \mathrm{Sb}_{7} \mathrm{~S}_{70}$ and $\mathrm{As}_{40} \mathrm{~S}_{60}$ chalcogenide glass films," J. Virtual Exp. 114, e54379 (2016).

87. S. Novak et al., "Deposition of $\mathrm{Ge}_{23} \mathrm{Sb}_{7} \mathrm{~S}_{70}$ chalcogenide glass films by electrospray," Thin Solid Films 588, 56 (2015).

88. Y. Zou, "Chalcogenide glasses for advanced photonic and photovoltaic applications," PhD Dissertation, University of Delaware (2015).

89. K. M. Davis et al., "Writing waveguides in glass with a femtosecond laser," Opt. Lett. 21, 1729 (1996).

90. A. Szameit et al., "Two-dimensional soliton in cubic fs laser written waveguide arrays in fused silica," Opt. Express 14, 6055 (2006).

91. O. M. Efimov et al., "High efficiency Bragg gratings in photothermal refractive glass," Appl. Opt. 38, 619 (1999).

92. G. Pfister, "Electronic properties of chalcogenide glasses and their use in xerography," J. Electron. Mater. 8, 789-837 (1979).

93. K. D. Kolwicz and M. S. Chang, "Silver halide-chalcogenide glass inorganic resists for X-ray lithography," J. Electrochem. Soc. 127, 135 (1980).

94. K. A. Richardson et al., "Comparison of nonlinear optical properties of sulfide glasses in bulk and thin film form," J. Opt. Mater. 10, 155 (1998).

95. N. Carlie et al., "Measurement of the refractive index dispersion of $\mathrm{As}_{2} \mathrm{Se}_{3}$ bulk glass and thin films prior to and after laser irradiation and annealing using prism coupling in the near- and mid-infrared spectral range," Rev. Sci. Instrum. 82, 053103 (2011).

96. A. V. Belykh et al., "Photo-structural transformation of chalcogenide glasses under nonlinear absorption of laser radiation," J. Non- Cryst. Solids 213, 330 (1997).

97. A. Schulte et al., "In-situ structural characterization of chalcogenide bulk-film-fiber properties by near-infrared waveguide Raman spectroscopy," Opt. Commun. 198, 125 (2001).

98. C. Rivero, A. Schulte, and K. A. Richardson, "Structure-property relationships in As-S-Se glasses for waveguide applications probed by waveguide Raman spectroscopy," Ceram. Trans. 126, 79 (2002).

99. J. D. Musgraves et al., "Comparison of the optical, thermal and structural properties of Ge-Sb-S thin films deposited using thermal evaporation and pulsed laser deposition techniques," Acta Mater. 59, 5032 (2011). 
100. K. Richardson et al., "Engineering glassy chalcogenide materials for integrated optics application," in Photo-Induced Metastability in Amorphous Semiconductors, A. V. Kolobov, Ed., Wiley-VCH Verlag \& Co., Weinheim (2003).

101. A. Saliminia et al., "Photo-induced Bragg reflectors in As-S-Se/As-S based chalcogenide glass multilayer channel waveguides," J. Fiber Integr. Opt. 20, 151 (2001).

102. L. Shah et al., "Femtosecond laser processing of glasses and polymers in air," Appl. Surf. Sci. 183, 151 (2001).

103. O. M. Efimov et al., "Waveguide writing in chalcogenide glasses by a train of femtosecond laser pulses," J. Opt. Mater. 17, 379 (2001).

104. A. Saliminia et al., "Temperature dependence of Bragg reflectors in chalcogenide $\mathrm{As}_{2} \mathrm{~S}_{3}$ glass slab waveguides," J. Opt. Soc. B 17, 1343 (2000).

105. K. A. Richardson et al., "Non-linear optical properties of chalcogenide glasses in the system As-S-Se," J. Non-Cryst. Solids 256, 353 (1999).

106. A. Saliminia et al., "First and second order Bragg gratings in single mode planar waveguides of chalcogenide glasses," J. Lightwave Technol. 17, 837 (1999).

107. J.-F. Viens et al., "Fabrication and characterization of integrated optical waveguides in arsenic (S,Se)-based chalcogenide glasses," J. Lightwave Technol. 17, 1184 (1999).

108. C. Meneghini et al., "Luminescence from Nd-ion implanted $\mathrm{As}_{2} \mathrm{~S}_{3}$ waveguides," J. Opt. Soc. B 15, 1305 (1997).

109. T. V. Galstian et al., "Photoinduced self-developing relief gratings in thin film chalcogenide $\mathrm{As}_{2} \mathrm{~S}_{3}$ glasses," J. Lightwave Technol. 15, 1343 (1997).

110. "Large-scale, Reconfigurable and Multifunctional 2.5-D Conformal Optics" funded by the Defense Advanced Research Projects Agency through the Defense Sciences Office (DSO) Program EXTREME Optics and Imaging (EXTREME) under Agreement No. HR00111720029.

111. C. M. Schwarz et al., "Processing and fabrication of micro-structures by multiphoton lithography in germanium-doped arsenic selenide," Opt. Mater. Express 8, 1902-1915 (2018).

112. P. McCarthy and D. T. Moore, "Optical design with gradient-index elements constrained to real material properties," in Opt. Fab. and Testing, Optical Society of America (2012).

113. R. A. Flynn et al., "Achromatic GRIN singlet lens design," Opt. Express 21, 4970 (2013).

114. S. D. Campbell et al., "On the use of surrogate models in the analytical decompositions of refractive index gradients obtained through quasiconformal transformation optics," J. Opt. 18, 044019 (2016).

115. K. L. Morgan et al., "Transformation-optics-inspired anti-reflective coating design for gradient index lenses," Opt. Lett. 40, 2521 (2015).

116. J. A. Easum et al., "Analytical surrogate model for the aberrations of an arbitrary GRIN lens," Opt. Express 24, 17805 (2016).

117. S. D. Campbell et al., "SWaP reduction regimes in achromatic GRIN singlets," Appl. Opt. 55, 3594 (2016).

118. S. D. Campbell, J. Nagar, and D. H. Werner, "Multi-element, multi-frequency lens transformations enabled by optical wavefront matching," Opt. Express 25, 17258 (2017).

119. A. M. Boyd, "Optical design of athermal, multispectral, radial gradient-index lenses," Opt. Eng. 57, 085103 (2018).

120. A. M. Boyd, "Optical design of athermal, multispectral, radia GRIN lenses," Proc. SPIE 10181, 1018109 (2017).

121. "SCHOTT glass technical information sheet - (TIE-26) homogeneity of optical glass," https://www.us.schott.com/d/advanced_optics/6dbf4e36-181c-4594-b1e1-a42d49a49cb7/ 1.2/schott_tie_26_homogeneity_of_optical_glass_us.pdf (accessed May 2020).

122. " $\mathrm{M}^{3}$ Measurement Solutions Inc," http://www.m3msi.com/m3-measurement-solutionsservices.html (accessed May 2020).

123. T. Binkele et al., "Characterization of gradient index optical components using experimental ray tracing," Proc. SPIE 10925, 109250D (2019).

124. G. P. Lindberg et al., "Methods of both destructive and non-destructive metrology of GRIN optical elements," Proc. SPIE 9451, 94511S (2015). 
125. J. Yao et al., "Volumetric rendering and metrology of spherical gradient refractive index lens imaged by angular scan optical coherence tomography system," Opt. Express 24, 19388 (2016).

126. "GRIN Group Metrology Instruments," http://www.optics.rochester.edu/workgroups/ moore/downloads (accessed May 2020).

127. A. Yee, "Mid-wave and long-wave infrared gradient-index optics: metrology, design, and athermalization," PhD Dissertation, University of Rochester Institute of Optics (2018).

128. A. J. Yee and D. T. Moore, "Optical design study in the 3-12 $\mu \mathrm{m}$ spectral band with gradient-index materials," Proc. SPIE 10590, 105901D (2017).

129. P. McCarthy et al., "Optical design study in the $1-5 \mu \mathrm{m}$ spectral band with gradient-index materials," Proc. SPIE 9293, 92930X (2014).

130. J. A. Corsetti, P. McCarthy, and D. T. Moore, "Color correction in the infrared using gradient-index materials," Opt. Eng. 52, 112109 (2013).

131. A. J. Yee et al., "Design of a freeform gradient-index prism for mixed reality head mounted display," Proc. SPIE 10676, 106760S (2018).

132. A. J. Yee et al., "The first order solutions for two configurations of discrete zoom lenses," Proc. SPIE 9822, 982215 (2016).

133. A. J. Yee et al., "New tools for finding first-order zoom lens solutions and the analysis of zoom lenses during the design process," Proc. SPIE 9580, 958006 (2015).

134. R. Berman et al., "Optical design study of a VIS-SWIR 3X zoom lens," Proc. SPIE 9580, 95800D (2015).

Kathleen A. Richardson is currently a Pegasus professor at CREOL, the College of Optics and Photonics at the University of Central Florida. Her research team carries out synthesis and characterization of chalcogenide glasses and glass-ceramics, examining the role of structureproperty relationships on optical performance in bulk, planar, and fiber materials. Her group has extensive industrial and government supported research developing materials for gradient refractive index optics, precision molded optics, and detection applications.

Myungkoo Kang is currently a research scientist at CREOL, the College of Optics and Photonics at the University of Central Florida. He received his $\mathrm{PhD}$ in materials science and engineering from the University of Michigan and worked as a postdoctoral associate in the Department of Electrical Engineering at the Pennsylvania State University. His research has utilized irradiation on chalcogenide glasses to create nanocomposites with spatially tunable microstructure and properties for gradient refractive index lenses.

Biographies of the other authors are not available. 